TRANSACTIONS OF THE

AMERICAN MATHEMATICAL SOCIETY

Volume 356, Number 2, Pages 427-456

S 0002-9947(03)03154- 4

Article electronically published on September 22, 2003

\title{
ASYMPTOTIC RELATIONS AMONG FOURIER COEFFICIENTS OF AUTOMORPHIC EIGENFUNCTIONS
}

\author{
SCOTT A. WOLPERT
}

\begin{abstract}
A detailed stationary phase analysis is presented for noncompact parameter ranges of the family of elementary eigenfunctions on the hyperbolic plane $\mathcal{K}(z)=y^{1 / 2} K_{i r}(2 \pi m y) e^{2 \pi i m x}, z=x+i y, \lambda=\frac{1}{4}+r^{2}$ the eigenvalue, $s=$ $2 \pi m \lambda^{-1 / 2}$ and $K_{i r}$ the Macdonald-Bessel function. The phase velocity of $\mathcal{K}$ on $\{|s| \operatorname{Im} z \leq 1\}$ is a double-valued vector field, the tangent field to the pencil of geodesics $\mathcal{G}$ tangent to the horocycle $\{|s| \operatorname{Im} z=1\}$. For $A \in S L(2 ; \mathbb{R})$ a multiterm stationary phase expansion is presented in $\lambda$ for $\mathcal{K}(A z) e^{2 \pi i n} \operatorname{Re} z$ uniform in parameters. An application is made to find an asymptotic relation for the Fourier coefficients of a family of automorphic eigenfunctions. In particular, for $\psi$ automorphic with coefficients $\left\{a_{n}\right\}$ and eigenvalue $\lambda$ it is shown for the special range $n \sim \lambda^{1 / 2}$ that $a_{n}$ is $O\left(\lambda^{1 / 4} e^{\pi \lambda^{1 / 2} / 2}\right)$ for $\lambda$ large, improving by an order of magnitude for this special range upon the estimate from the general Hecke bound $O\left(|n|^{1 / 2} \lambda^{1 / 4} e^{\pi \lambda^{1 / 2} / 2}\right)$. An exposition of the WKB asymptotics of the Macdonald-Bessel functions is presented.
\end{abstract}

\section{INTRODUCTION}

A cuspidal automorphic eigenfunction for the hyperbolic Laplacian has a Fourier series expansion

$$
\psi(z)=\sum_{n \neq 0} a(n) y^{1 / 2} K_{i r}(2 \pi|n| y) e^{2 \pi i n x}
$$

for $z=x+i y, y>0$, eigenvalue $-\lambda=-\left(\frac{1}{4}+r^{2}\right)<-1 / 4$ and $K_{i r}$ a MacdonaldBessel function, [Iw2 $\mathrm{Vk}, \mathrm{Tr}$. The Fourier coefficients are of interest, $\mathrm{Bm}, \mathrm{BDHI}$ HA Iw1 Iw2, Ms, Pt1, Pt2, Tr, Sr1, Sb1 Vk. For instance for $\Gamma=S L(2 ; \mathbb{Z})$ and the Hecke basis of eigenfunctions, the Hecke-Maass $L$-series $L(s, \psi)=\sum_{n=1}^{\infty} a(n) n^{-s}$ continues to an entire function of $s$ with an Euler product

$$
L(s, \psi)=\prod_{p}\left(1-a(p) p^{-s}+p^{-2 s}\right)^{-1}
$$

for $\operatorname{Re} s>1$, and for $\psi$ even a functional equation $\Lambda(s, \psi)=\Lambda(1-s, \psi)$ for $\Lambda(s, \psi)=\pi^{-s} \Gamma\left(\frac{s+i r}{2}\right) \Gamma\left(\frac{s-i r}{2}\right) L(s, \psi), \Gamma(z)$ the Euler gamma function, $\mathrm{Bm}$, GSh].

Received by the editors December 15, 1999 and, in revised form, October 13, 2000

2000 Mathematics Subject Classification. Primary 11F30, 33C10; Secondary 11M06, 42A16.

Key words and phrases. Automorphic eigenfunctions, Macdonald-Bessel functions, Fourier coefficients.

This research was supported in part by NSF Grants DMS-9504176 and DMS-9800701. 
The order of magnitude of the Fourier coefficients and their sums are basic issues, even for nonarithmetic groups [DI, Iw2, Pt1, [Pt2, [Sr1, Sb2]. Hecke gave the argument for the elementary bound valid for $\Gamma$ cofinite with a cusp at infinity $|a(n)| e^{-\pi \lambda^{1 / 2} / 2} \leq C_{\Gamma}|n|^{1 / 2} \lambda^{1 / 4}$ (the factor $e^{\pi \lambda^{1 / 2} / 2}$ is an artifact of the normalization of the Macdonald-Bessel functions), $\mathrm{Tr}$. The magnitude is also a basic matter for questions in quantum chaos, [Sr1, Wp. Numerical investigations of D. A. Hejhal [HA, $\mathrm{Hj} 2$ have provided evidence for a generalized Ramanujan-Petersson conjecture: the coefficients $|a(n)|$ should be $O_{\lambda, \epsilon}\left(|n|^{\epsilon}\right)$.

The focus of the present investigation is a set of relations among the Fourier coefficients of a cuspidal automorphic eigenfunction. To present the relations we first introduce the required quantities. Let $h(\mu)$ be a smooth function with $\operatorname{supp}(h) \subset$ $(0,2)$ and $\sum_{n \in \mathbf{Z}} h(\mu+n) \equiv 1$. For $n, m \in \mathbb{Z}$ and $A=\left(\begin{array}{ll}a & b \\ c & d\end{array}\right) \in \Gamma, c>0$, introduce the parameters $t=2 \pi n \lambda^{-1 / 2}, s=2 \pi m \lambda^{-1 / 2}$ and $\tau=c^{2}|s t|^{-1}$. Further define the (phase) function $Q_{0}=2\left(1+\tau^{-1}\right)^{1 / 2}-\log \left(2 \tau+1+2\left(\tau^{2}+\tau\right)^{1 / 2}\right)-(t d+s a) c^{-1}$. We find in Corollary 4.9 that, given parameter values $0<t_{0}<t_{1}$ and $\beta>4$, the Fourier coefficients $\left\{a_{n}\right\}$ of an automorphic eigenfunction $\psi$ with eigenvalue $\lambda$ satisfy

$$
\begin{gathered}
a_{n} e^{-\pi \lambda^{1 / 2} / 2}=\lambda^{-1 / 4} \sum_{m>0} a_{m} e^{-\pi \lambda^{1 / 2} / 2} e^{-i \lambda^{1 / 2} Q_{0}} \sum_{k=0}^{4} \lambda^{-k / 6} g_{k}(t, s) \\
+O\left(\lambda^{-7 / 12}\|\psi\|_{2}\right)
\end{gathered}
$$

for $t_{0} \leq 2 \pi n \lambda^{-1 / 2} \leq t_{1}$ and a remainder constant depending on $t_{0}, t_{1}, \beta, h, A$ and $\Gamma$. We find that the Fourier coefficients $a_{n}$, for the hybrid range $t_{0} \leq 2 \pi n \lambda^{-1 / 2} \leq t_{1}$, are bounded as $O\left(\lambda^{1 / 4} e^{\pi \lambda^{1 / 2} / 2}\|\psi\|_{2}\right)$. The present bound for the hybrid range is a considerable improvement over the estimate obtained by specializing the general bounds of P. Sarnak [Sr2] and Y. N. Petridis [Pt1, Pt2]. Petridis has established the general bound that $a_{n}$ is $O\left(|n|^{3 / 8+\epsilon} \lambda^{1 / 4} e^{\pi \lambda^{1 / 2} / 2}\|\psi\|_{2}\right)$. Each Fourier coefficient is presented above as a sum of Fourier coefficients with the phase $-\lambda^{1 / 2} Q_{0}$. The coefficients $g_{k}$ are smooth functions of $t, s$ and are supported on the interval $\beta<$ $\tau^{-1}+2<\alpha, \alpha=\left(2 t_{1}+(\beta-1)^{1 / 2}\right)^{2}$ (see (19) and Section 4.7 for the definition of the coefficients); the first coefficient is

$$
\begin{aligned}
g_{0}= & (\pi / 2)^{1 / 2} c^{-1} e^{-i \pi / 4} \tau^{1 / 2}(2 \tau+1)^{2 / 3}\left(\tau^{2}+\tau\right)^{-5 / 12} \\
& \times h\left(|t|^{-1}\left(\left(\tau^{-1}+1\right)^{1 / 2}-(\beta-1)^{1 / 2}\right)\right) .
\end{aligned}
$$

The sum over $m$ is in fact supported in the range

$$
c^{2} t^{-1}(\beta-2)<2 \pi m \lambda^{-1 / 2}<c^{2} t^{-1}(\alpha-2) .
$$

The focus of our approach is the stationary phase expansion in the eigenvalue $\lambda=\frac{1}{4}+r^{2}$ of the integral

$$
\begin{aligned}
\int_{\operatorname{Im} w=\lambda^{1 / 2}(2 \pi|n|)^{-1}}(\operatorname{Im} A(w))^{1 / 2} K_{i r}(2 \pi \mid & m \mid \operatorname{Im} A(w)) \\
\times & e^{2 \pi i m \operatorname{ReA} A(w)+2 \pi i n \operatorname{Rew}} \tilde{h}(\operatorname{Rew}) d \operatorname{Rew}
\end{aligned}
$$


for $m, n \in \mathbb{Z}, A \in S L(2 ; \mathbb{R})$ and $\tilde{h}$ a suitable test function. We start our considerations in Section 2 by recalling the Liouville-Green asymptotics of the MacdonaldBessel functions

$$
\begin{gathered}
2 e^{\pi \lambda^{1 / 2} / 2}(|m| \operatorname{Im} z)^{1 / 2} K_{i r}(2 \pi|m| \operatorname{Im} z) e^{2 \pi i m \operatorname{Re} z} \\
=(|s| \operatorname{Im} z)^{1 / 2}\left(e^{i \lambda^{1 / 2} \Psi_{+}(z)-i \pi / 4}+e^{i \lambda^{1 / 2} \Psi_{-}(z)+i \pi / 4}\right)+O\left(\lambda^{-1 / 2}\right)
\end{gathered}
$$

for $s=2 \pi m \lambda^{-1 / 2},|s| \operatorname{Im} z<<1$ and the phase function $\Psi_{ \pm}(z)=s \operatorname{Re} z \pm F(|s| \operatorname{Im} z)$ for $\left.F(Y)=\int_{Y}^{1}\left(\tau^{-2}-1\right)^{1 / 2} d \tau,[\mathrm{Bl}] \mathrm{Ov}\right]$. We find that the hyperbolic gradient $\nabla \Psi_{ \pm}$, the phase velocity, is a double-valued vector field on $\{|s| \operatorname{Imz} \leq 1\}$ with a simple geometric description. If $\mathcal{G}$ is the pencil of geodesics tangent to the horocycle $\{|s| \operatorname{Im} z=1\}$, then $\nabla \Psi_{ \pm}$is the rightward pointing unit tangent double-field to $\mathcal{G}$ (see Figure 2). The graph of the differential $d \Psi_{ \pm}$gives a two-sheeted Lagrangian over $\{|s| \operatorname{Im} z<1\}$ which folds over $\{|s| \operatorname{Im} z=1\}$. Similarly the phase of the integrand of (3) is $\Phi=\epsilon_{*} F(|s| \operatorname{Im} A(w))+\operatorname{Re}(s A(w)+t w)$ for $\epsilon_{*}= \pm 1, s=2 \pi m \lambda^{-1 / 2}$ and $t=2 \pi n \lambda^{-1 / 2}$. In Section 3.2 we show that on the horocycle $\{|t| \operatorname{Imw}=1\}$ the phase derivative is given as

$$
\frac{d \Phi}{d \operatorname{Rew}}=t\left(\left(\left(1+\epsilon_{1} \rho\right) \rho \tau\right)^{1 / 2}+\epsilon_{2}\left((1-\rho \tau)\left(1-\epsilon_{1} \rho\right)\right)^{1 / 2}\right)^{2}
$$

for the parameters $A=\left(\begin{array}{cc}* & * \\ c & *\end{array}\right), \tau=c^{2}|s t|^{-1}, \rho=\left|s t^{-1}\right|\left|A^{\prime}(w)\right|, \epsilon_{1}=\operatorname{sgn}(-s t)$ and $\epsilon_{2}=\operatorname{sgn}\left(\epsilon_{*} t\right)$. It follows directly that the phase $\Phi$ is stationary (to second order) precisely for $\epsilon_{2}=-1$ and $\rho=\left(2 \tau+\epsilon_{1}\right)^{-1}$.

In Section 4 (see Propositions 4.2 through 4.7) we use the explicit description of the Macdonald-Bessel function asymptotics, the phase $\Phi$ and its derivative $\frac{d \Phi}{d R e w}$ to give a five-term stationary phase expansion of the integral (3) for a range of the $t$ and $s$ parameter values. The expansion is the focus of this paper.

To obtain the coefficient relation (2) for an automorphic eigenfunction $\psi$, consider for $A \in \Gamma$ the integrals

$$
\begin{aligned}
& \int_{|t| I m w=1} \psi(w) e^{2 \pi i n R e w} \tilde{h}(\text { Rew }) d R e w \\
& =\int_{|t| I m w=1} \psi(A(w)) e^{2 \pi i n \operatorname{Rew}} \tilde{h}(\operatorname{Rew}) d \operatorname{Rew}
\end{aligned}
$$

and insert the series representation (1) of $\psi$ on both the left and right sides. The right-hand side is a sum of products of Fourier coefficients of $\psi$ and integrals (3). This is the fundamental coefficient relation; the full statement and proof are given in Theorem 4.8 Provided $\sum_{n \in \mathbb{Z}} \tilde{h}(x+n) \equiv 1$, by the orthogonality of exponentials the left-hand side reduces to a multiple of the single coefficient $a_{-n},|t|=2 \pi|n| \lambda^{-1 / 2}$, and we find the stated relation.

A cuspidal automorphic eigenfunction $\psi$ with eigenvalue $\lambda$ gives rise to linear forms

$$
\mathcal{L}_{k_{0}, k_{1}}(\psi)=\sum_{k_{0} \leq k \leq k_{1}} a_{k} e^{2 \pi i k \nu}
$$


The question of analyzing the signs of the Fourier coefficients has previously been studied following E. Hecke with the individual bound that $\mathcal{L}_{k_{0}, k_{1}}(\psi)$ is

$$
O_{\epsilon}\left(\left(\lambda k_{1}\right)^{1 / 2+\epsilon} e^{\pi \lambda^{1 / 2} / 2}\|\psi\|_{2}\right)
$$

(see the discussion in Section 8.3 of [w2] and in Section 4 of [w1) and with spectral averaging in the work of Deshouillers and Iwaniec, [DI] A heuristic model from analytic number theory suggests that the coefficients $\left\{a_{n} e^{2 \pi i n \nu} e^{\pi \lambda^{1 / 2} / 2}\right\}$ could behave as independent identically distributed random variables with variance comparable to unity. In particular, for $k_{0}$ and $k_{1}-k_{0}$ substantial the linear form $\mathcal{L}_{k_{0}, k_{1}}(\psi)$ would have magnitude

$$
O_{\epsilon}\left(\left(k_{1}-k_{0}\right)^{1 / 2+\epsilon} \lambda^{\epsilon} e^{\pi \lambda^{1 / 2} / 2}\|\psi\|_{2}\right) .
$$

Similarly, for $\left\{a_{n} e^{2 \pi i n \nu} e^{-i \lambda^{1 / 2} Q_{0}} e^{\pi \lambda^{1 / 2} / 2}\right\}$ independent identically distributed random variables the right-hand side of the above relation would have magnitude $O_{h}\left(\lambda^{\epsilon}\|\psi\|_{2}\right)$ in accordance with the generalized Ramanujan-Petersson conjecture. In $\overline{\mathrm{Wp}}$ we presented a formula for the microlocal lift of $\psi$ to $S L(2 ; \mathbf{R})$ with $\lambda$ large in terms of the linear forms $\mathcal{L}_{k_{0}, k_{1}}(\psi)$. We found that the microlocal lift of $\psi$ approximates a constant (the wave $\psi$ and its derivatives are uniformly distributed) if and only if $\left|\mathcal{L}_{k_{0}, k_{1}}(\psi)\right|^{2}$ weak $^{*}$ approximates a constant as a function of $\nu$ and the sum length $\left(k_{1}-k_{0}\right) \lambda^{-1 / 2}$. In Section 4.8 we find that the linear forms also occur as the leading term in the expansion in $\lambda$ for the integral of an eigenfunction over a vertical geodesic in the upper half plane.

Our original purpose was to use the automorphy condition to derive Fourier coefficient relations, which in turn could be used to determine the behavior of the coefficients and their sums. The status is as follows. A unit-norm automorphic eigenfunction has coefficients with average square magnitude unity; a slight improvement of the standard bound is presented in the Appendix. The right-hand side of the relation (2) in general will have at least unit magnitude while the provided remainder term is significantly smaller, with magnitude $O\left(\lambda^{-7 / 12}\right)$. The relation in effect provides a matrix equation for the vector of Fourier coefficients of length $\lambda^{1 / 2}$, one equation for each element of the discrete group. The relation provides an immediate restricted bound for $a_{n}$ without analyzing cancellation in coefficient sums. The relation appears to provide no special information on the cancellation. An open matter is to find a tractable transformation of the coefficient sums.

I would like to thank Wenzhi Luo, Peter Sarnak and Steven Zelditch for their valued suggestions. I would also like to thank the Institute for Physical Science and Technology at the University of Maryland, the Graduate Research Board of the University of Maryland, as well as the Institute for Mathematics and its Applications at the University of Minnesota.

\section{The Liouville-Green approximation FOR THE MACDONALD-BESSEL FUnCTIONS}

2.1. The Liouville-Green approximation (WKB) is a well established prescription for finding the asymptotics of solutions of differential equations Ov, esp. Chaps. 6 and 11]. R. E. Langer refined the method to give uniform asymptotic solutions to turning point problems, $[\mathrm{Ov}]$. For the Macdonald-Bessel functions the approximation is given in terms of two scalings, a change of variables, and the Airy function. 
The consequence is that the asymptotics of $y^{1 / 2} K_{i r}(y)$ in $y$ and $\lambda=\frac{1}{4}+r^{2}$ are given by the asymptotics of a single function $A i(u)$. The required Liouville-Green approximation is given in Chapter 11, Sections 3 and 7 of $[\mathrm{Ov}]$. The approximation for the Macdonald-Bessel functions is given in Chapter 11, Section 10 of [Ov], and also by C. Balogh in Section 3 of [B]. The prescription will be reviewed in the following sections. The basic change of variables is presented in Section 2.2 The approximating solution is presented in Definition 2.1 and the error bound in Theorem 2.2. In Section 2.4 we review the asymptotics of the approximating solution and consider first consequences. In Section 2.5 we review the stationary phase expansion for a cubic phase, and also provide the stationary phase expansion for the Macdonald-Bessel function.

2.2. We start with the differential equation

$$
K^{\prime \prime}(y)=\left(L^{2}-\lambda y^{-2}\right) K(y), \quad y>0, \lambda>0,
$$

for the Macdonald-Bessel functions ( $K$-Bessel functions), [Lb, Sec 5.7]. The rescaled independent variable is $Y=t y$ for $t^{2}=L^{2} \lambda^{-1}, t>0$. The composition $K T(Y)=$ $K\left(Y t^{-1}\right)$ satisfies

$$
K T^{\prime \prime}(Y)=\lambda\left(1-Y^{-2}\right) K T(Y)
$$

as a function of $Y$. The factor $\left(1-Y^{-2}\right)$ will play a basic role in this section and the next. The differential equation is now in the standard form given in $\mathrm{Ov}$ Chap. 11, Subsection 3.1, display (3.01)] with $u^{2}=\lambda, f=\left(1-Y^{-2}\right)$ and $g \equiv 0$.

We next consider the important change of variables $\zeta(Y)$ defined by

$$
\begin{aligned}
\frac{2}{3}(-\zeta)^{3 / 2} & =\int_{Y}^{1}\left(\tau^{-2}-1\right)^{1 / 2} d \tau \text { for } 0<Y \leq 1 \\
& =-\left(1-Y^{2}\right)^{1 / 2}+\log \left(\left(1+\left(1-Y^{2}\right)^{1 / 2}\right) / Y\right) \\
\frac{2}{3}(\zeta)^{3 / 2} & =\int_{1}^{Y}\left(1-\tau^{-2}\right)^{1 / 2} d \tau \text { for } Y \geq 1 \\
& =\left(Y^{2}-1\right)^{1 / 2}+\arcsin \left(Y^{-1}\right)-\pi / 2
\end{aligned}
$$

(Balogh and Olver write $z$ in place of $Y$, and thus $\zeta(z)$ ). The function $\zeta(Y)$ is real analytic on $\mathbb{R}^{+}$with positive derivative, satisfies the defining relation $\zeta\left(\frac{d \zeta}{d Y}\right)^{2}=$ $\left(1-Y^{-2}\right)$ and has the following expansions:

$$
\begin{aligned}
\frac{2}{3}(-\zeta)^{3 / 2} & =\log (2 / Y)-1+O\left(Y^{2}\right) \text { for } Y \text { near } 0, \\
Y & =2 e^{-2 / 3(-\zeta)^{3 / 2}-1}+O\left(e^{-2(-\zeta)^{3 / 2}}\right) \text { for } \zeta \text { large negative, } \\
\frac{2}{3}(-\zeta)^{3 / 2} & =\frac{1}{3}\left(1-Y^{2}\right)^{3 / 2}+O\left((Y-1)^{2}\right) \text { for } Y \text { near } 1, Y \leq 1, \\
\frac{2}{3}(\zeta)^{3 / 2} & =Y-\pi / 2+O\left(Y^{-1}\right) \text { for } Y \text { large positive, } \\
Y & =\frac{2}{3}(\zeta)^{3 / 2}+\pi / 2+O\left(\zeta^{-3 / 2}\right) \text { for } \zeta \text { large positive. }
\end{aligned}
$$$$
\text { and }
$$ 


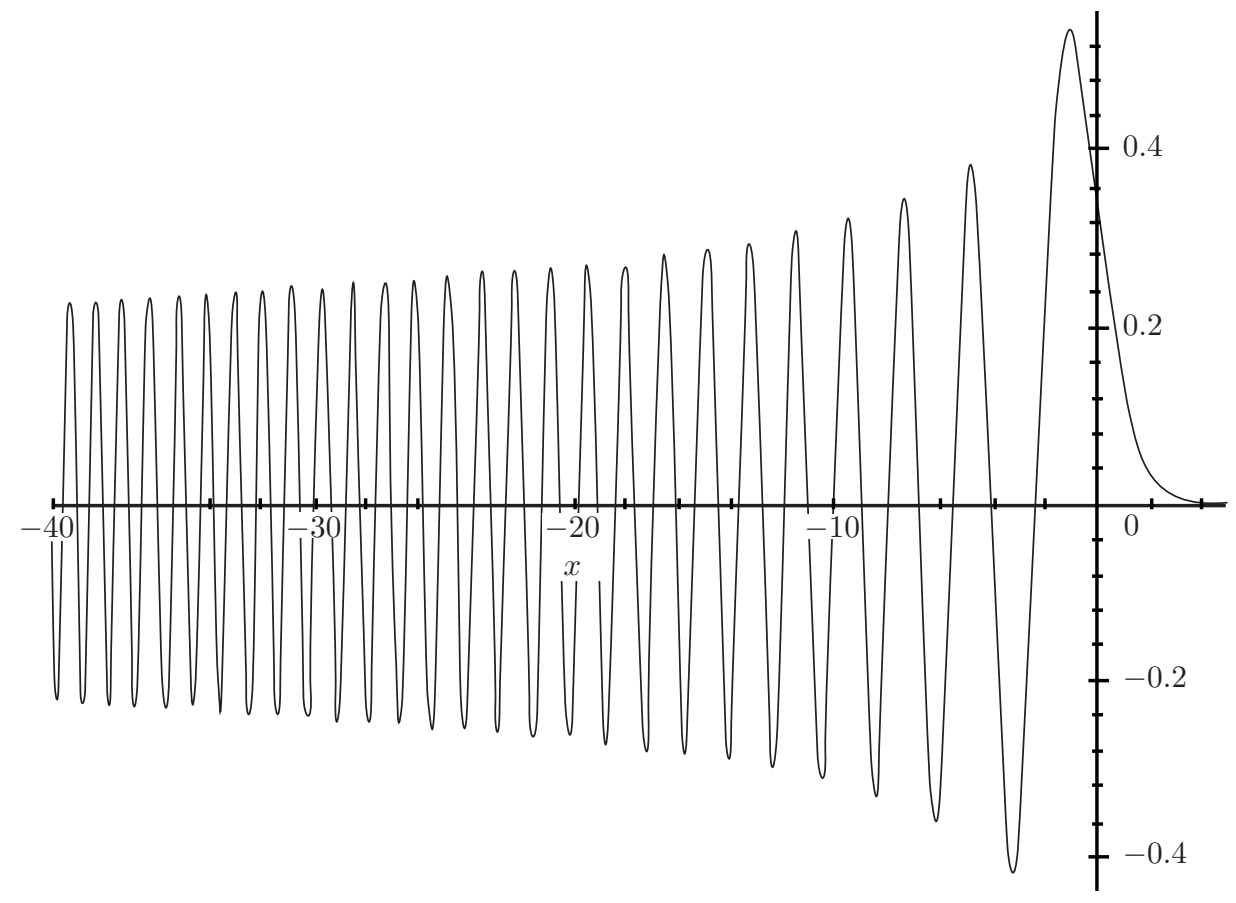

Figure 1. The Airy function $A i(x)$

2.3. We present the Liouville-Green model solution (the approximating solution) to (5), as well as the error estimate. The change of variables $\zeta$ gives the standard form for the differential equation $[\mathrm{Ov}$, pg. 398, esp. display (3.04)] (we write $Y(\zeta)$ for the inverse function).

Definition 2.1. The model solution is, for $Y>0$,

$$
M S(Y)=\left(\zeta(Y) /\left(1-Y^{-2}\right)\right)^{1 / 4}\left(A i\left(\lambda^{1 / 3} \zeta(Y)\right)+\lambda^{-2 / 3} B_{0}(\zeta(Y)) A i^{\prime}\left(\lambda^{1 / 3} \zeta(Y)\right)\right)
$$

where

$$
B_{0}(\zeta)=-\frac{5}{48} \zeta^{-2}+\frac{1}{24}\left(\left(Y(\zeta)^{2}-1\right) \zeta\right)^{-1 / 2}\left(3+5\left(Y(\zeta)^{2}-1\right)^{-1}\right) .
$$

Airy's function of the first kind is

$$
\operatorname{Ai}(\mu)=\frac{1}{\pi} \int_{0}^{\infty} \cos \left(\frac{1}{3} \tau^{3}+\mu \tau\right) d \tau
$$

[Lb, Sec. 5.17], Ov, Chaps. 2 and 11]; the basic properties of $A i$ will be cited. The first is that $A i$ is a solution of the differential equation $A^{\prime \prime}(\mu)=\mu A(\mu)$. A graph of $A i(\mu)$ is given in Figure 1, the Airy function is a library function for Maple V.

Now from Theorems 3.1 and 7.1 of Chapter 11 of Olver the above differential equation (5) has a solution, denoted $w_{2}$ in Theorem 3.1 and $W_{3,2}$ in Theorem 7.1, with

$$
W_{3,2}=M S(Y)+\left(\zeta(Y) /\left(1-Y^{-2}\right)\right)^{1 / 4}\left(\lambda^{-1} A_{1}(\zeta(Y)) A i\left(\lambda^{1 / 3} \zeta(Y)\right)+\epsilon_{3,2}\right),
$$


where from [B] Sec. 3] and $\mathrm{Ov}$ Chap. 11, Subsection 7.2]

$$
\begin{gathered}
\psi(\zeta)=\frac{5}{16} \zeta^{-2}-\frac{1}{4} \zeta Y(\zeta)^{2}\left(Y(\zeta)^{2}+4\right)\left(Y(\zeta)^{2}-1\right)^{-3}, \\
2 \zeta^{1 / 2} B_{0}(\zeta)=\int_{0}^{\zeta} \psi(\mu) \mu^{-1 / 2} d \mu
\end{gathered}
$$

(the square roots are given by the same branch)

$$
\begin{gathered}
2 A_{1}(\zeta)=-B_{0}^{\prime}(\zeta)+\int \psi(\zeta) B_{0}(\zeta) d \zeta \\
2 \zeta^{1 / 2} B_{1}(\zeta)=\int_{0}^{\zeta}\left(\psi(\mu) A_{1}(\mu)-A_{1}^{\prime \prime}(\mu)\right) \mu^{-1 / 2} d \mu,
\end{gathered}
$$

and

$$
\begin{aligned}
& \left|\epsilon_{3,2}\right| \leq \\
& 2 \lambda^{-3 / 2} \frac{M}{E}\left(\lambda^{1 / 3} \zeta(Y)\right) \exp \left(2 a \lambda^{-1 / 2} \mathcal{V}_{\zeta(Y), \infty}\left(|\mu|^{1 / 2} B_{0}(\mu)\right)\right) \mathcal{V}_{\zeta(Y), \infty}\left(|\mu|^{1 / 2} B_{1}(\mu)\right) .
\end{aligned}
$$

Clarifications are needed. The functions $\psi, B_{0}, B_{1}, A_{0}$ and $A_{1}$ are real analytic; the constant $a$ (Olver's $\lambda$ ) is explicit $(a \approx 1.04) ; \mathcal{V}_{\alpha, \beta}$ denotes the absolute variation on the interval $[\alpha, \beta] . \quad M$ and $E$ are explicit functions with the quotient $\frac{M}{E}(\mu)$, hence notated $M E(\mu)$, an explicit envelope for the Airy function, discussed in detail in $\mathrm{Ov}$, Chap. 11, Sec. 2]. Its basic properties are as follows: $M E$ is a continuous function on the real line with $M E(\mu) \sim(2 \pi)^{-1 / 2} \mu^{-1 / 4} e^{-2 / 3 \mu^{3 / 2}}$ as $\mu$ tends to positive infinity and $M E(\mu) \sim \pi^{-1 / 2}|\mu|^{-1 / 4}$ as $\mu$ tends to negative infinity. It is essential to understand the absolute variation $\mathcal{V}$. The Liouville-Green remainder involves $\mathcal{V}\left(|\mu|^{1 / 2} B_{0}(\mu)\right)$ and $\mathcal{V}\left(|\mu|^{1 / 2} B_{1}(\mu)\right.$ ). From the defining relation the absolute variation $\mathcal{V}_{-\infty, \infty}\left(|\mu|^{1 / 2} B_{0}(\mu)\right)$ is the $L^{1}(\mathbb{R})$-norm of $|\mu|^{-1 / 2} \psi(\mu)$. Now from the asymptotics of $Y(\zeta)$ it follows that $\psi(\zeta)$ and $B_{0}(\zeta)$ are both bounded by a multiple of $(1+|\zeta|)^{-2}$ for all $\zeta$. In particular, the $L^{1}(\mathbb{R})$-norm of $|\mu|^{-1 / 2} \psi(\mu)$ is finite. We are ready to consider $\mathcal{V}\left(|\mu|^{1 / 2} B_{1}(\mu)\right)$. From the defining relation for $\frac{d \zeta}{d Y}$ and an induction argument it follows that $\frac{d^{n} Y}{d \zeta^{n}}$ is $O_{n}\left(\zeta^{3 / 2-n}\right)$ for $\zeta$ large positive and is $O_{n, \epsilon}\left(e^{(-2 / 3+\epsilon)(-\zeta)^{3 / 2}}\right)$ for $\zeta$ large negative. It follows readily that $A_{1}$ is bounded and that $A_{1}^{\prime \prime}(\mu)$ is bounded by a multiple of $(1+|\mu|)^{-5}$. Now the absolute variation of $|\mu|^{1 / 2} B_{1}(\mu)$ on $\mathbb{R}$ is the $L^{1}(\mathbb{R})$-norm of $\left(\psi(\mu) A_{1}(\mu)-A_{1}^{\prime \prime}(\mu)\right)|\mu|^{-1 / 2}$, which in turn is bounded by a multiple of $(1+|\mu|)^{-2}|\mu|^{-1 / 2}$. The absolute variation $\mathcal{V}_{-\infty, \infty}\left(|\mu|^{1 / 2} B_{1}(\mu)\right)$ is finite.

With the above estimates we have a specific form of Balogh's and Olver's expansion [Bl, display (15)], Ov, Chap. 11, Sec. 10].

Theorem 2.2. The differential equation (5) has a solution

$$
\begin{aligned}
\mathcal{W}(Y)=\left(\zeta(Y) /\left(1-Y^{-2}\right)\right)^{1 / 4}\left(A i\left(\lambda^{1 / 3} \zeta(Y)\right)\right. & \\
& \left.+\lambda^{-2 / 3} B_{0}(\zeta(Y)) A i^{\prime}\left(\lambda^{1 / 3} \zeta(Y)\right)+\tilde{\epsilon}(Y)\right)
\end{aligned}
$$

for $Y=t y, t^{2}=L^{2} \lambda^{-1}, \zeta(Y)$ given in (6) and $B_{0}(\zeta)$ given in Definition 2.1. The remainder $\tilde{\epsilon}(Y)$ is bounded for $\lambda \geq 1$ by a multiple of $\lambda^{-1} M E\left(\lambda^{1 / 3} \zeta(Y)\right)$ for $M E$ the envelope for the Airy function $\left(M E(\mu) \sim(2 \pi)^{-1 / 2} \mu^{-1 / 4} e^{-2 / 3 \mu^{3 / 2}}\right.$, $\mu$ large positive; $M E(\mu) \sim \pi^{-1 / 2}|\mu|^{-1 / 4}$, $\mu$ large negative). 
2.4. We are ready to consider the basic behavior of the Airy function $\mathrm{Ov}$, Chap. 11, Sec. 1], [Lb, Sec. 5.17], and to relate the solution $\mathcal{W}$ of (5) to the MacdonaldBessel functions.

For $\mu$ tending to negative infinity

$$
\begin{aligned}
A i(\mu)= & \pi^{-1 / 2}|\mu|^{-1 / 4}\left(\cos \left(\frac{2}{3}|\mu|^{3 / 2}-\frac{\pi}{4}\right)\right. \\
& \left.+\frac{5}{48}|\mu|^{-3 / 2} \sin \left(\frac{2}{3}|\mu|^{3 / 2}-\frac{\pi}{4}\right)+O\left(|\mu|^{-3}\right)\right), \\
A i^{\prime}(\mu)= & \pi^{-1 / 2}|\mu|^{1 / 4}\left(\sin \left(\frac{2}{3}|\mu|^{3 / 2}-\frac{\pi}{4}\right)+O\left(|\mu|^{-3 / 2}\right)\right),
\end{aligned}
$$

and thus on also noting that $\left(\frac{5}{48}|\zeta|^{-3 / 2}+|\zeta|^{1 / 2} B_{0}(\zeta)\right)=-\frac{1}{24}\left(1-Y^{2}\right)^{-3 / 2}\left(3 Y^{2}+2\right)$ we have, as $Y$ approaches zero,

$$
\begin{aligned}
& M S(Y)=\lambda^{-1 / 12} \pi^{-1 / 2}\left(Y^{-2}-1\right)^{-1 / 4}\left(\cos \left(\frac{2}{3} \lambda^{1 / 2}|\zeta|^{3 / 2}-\frac{\pi}{4}\right)\right. \\
& \left.-\frac{\lambda^{-1 / 2}}{24}\left(1-Y^{2}\right)^{-3 / 2}\left(3 Y^{2}+2\right) \sin \left(\frac{2}{3} \lambda^{1 / 2}|\zeta|^{3 / 2}-\frac{\pi}{4}\right)\right) \\
& +O\left(\lambda^{-13 / 12} Y^{1 / 2}|\zeta|^{-3}\right) .
\end{aligned}
$$

For $\mu$ tending to positive infinity we have

$$
\begin{aligned}
A i(\mu) & =\left(2 \pi^{1 / 2} \mu^{1 / 4}\right)^{-1} e^{-2 \mu^{3 / 2} / 3}\left(1-\frac{5}{48} \mu^{-3 / 2}+O\left(\mu^{-3}\right)\right), \\
A i^{\prime}(\mu) & =\left(2 \pi^{1 / 2}\right)^{-1} \mu^{1 / 4} e^{-2 \mu^{3 / 2} / 3}\left(1+O\left(\mu^{-3 / 2}\right)\right)
\end{aligned}
$$

and thus, as $Y$ approaches positive infinity,

$$
\begin{aligned}
M S(Y) & =\lambda^{-1 / 12}\left(2 \pi^{1 / 2}\right)^{-1}\left(1-Y^{-2}\right)^{-1 / 4} e^{-2 \lambda^{1 / 2} \zeta^{3 / 2} / 3} \\
\times & \left(1+\lambda^{-1 / 2}\left(-\frac{5}{48} \zeta^{-3 / 2}+\zeta^{1 / 2} B_{0}(\zeta)\right)\right)+O\left(\lambda^{-13 / 12} e^{-2 \lambda^{1 / 2} \zeta^{3 / 2} / 3} \zeta^{-3}\right)
\end{aligned}
$$

and, given the asymptotics of $\zeta$ as $Y$ approaches infinity,

$$
\begin{gathered}
M S(Y)=\left(2 \pi^{1 / 2} \lambda^{1 / 12}\right)^{-1}\left(1-Y^{-2}\right)^{-1 / 4} e^{-\lambda^{1 / 2}\left(Y-\pi / 2+O\left(Y^{-1}\right)\right)} \\
\times\left(1+\lambda^{-1 / 2}\left(-\frac{5}{48} \zeta^{-3 / 2}+\zeta^{1 / 2} B_{0}(\zeta)\right)+O\left(\lambda^{-1} Y^{-2}\right)\right) .
\end{gathered}
$$

There is an important qualitative change in behavior of the Airy function $\operatorname{Ai}(\mu)$ at $\mu=0$, and of the model solution and Macdonald-Bessel function at $Y=1$. For values of the argument less than the turning point the functions are oscillatory, while for values greater than the turning point the functions have exponential decay. For example the model solution $M S$ is trigonometric in $\frac{2}{3} \lambda^{1 / 2} \zeta^{3 / 2}$ (with a $Y^{1 / 2}$ envelope) for $Y<1$, and exponential in $-\frac{2}{3} \lambda^{1 / 2} \zeta^{3 / 2}$ for $Y>1(\zeta>0)$.

The Macdonald-Bessel functions give rise to solutions of the differential equation (4). In particular, for $\lambda>1 / 4, \lambda=\frac{1}{4}+r^{2}$, then $y^{1 / 2} K_{i r}(|L| y)$ gives a solution to (4), vanishing at infinity, for $K_{i r}$ the Macdonald-Bessel function, [Lb Sec. 5.7].

Lemma 2.3. Notation as above. The Macdonald-Bessel function and solution $\mathcal{W}$ of (5i) are related by

$$
y^{1 / 2} K_{i r}(|L| y)=|L|^{-1 / 2} 2^{1 / 2} \pi \lambda^{1 / 12}\left(1+O\left(\lambda^{-1}\right)\right) e^{-\pi \lambda^{1 / 2} / 2} \mathcal{W}(Y) .
$$


Proof. A second solution $y^{1 / 2} I_{i r}(|L| y)$ to (4) is given in terms of the $I$-Bessel function. The second solution is unbounded for large $y$; all solutions to (4) bounded for large $y$ are multiples of $y^{1 / 2} K_{i r}(|L| y)$. It will suffice to consider the $y$-asymptotic expansion. For $L$ and $\lambda$ fixed, $y^{1 / 2} K_{i r}(|L| y)=(\pi / 2|L|)^{1 / 2} e^{-|L| y}\left(1+O\left(y^{-1}\right)\right)$, [Lb] pg. 123]. Similarly for $L$ and $\lambda$ fixed, given $|L| y=\lambda^{1 / 2} Y$, Theorem [2.2 (10) and that $B_{0}(\zeta)$ is $O\left(\zeta^{-2}\right)$ for $\zeta$ large, we have

$$
\mathcal{W}=\left(2 \pi^{1 / 2} \lambda^{1 / 12}\right)^{-1} e^{\pi \lambda^{1 / 2} / 2-|L| y}\left(1+O\left(\lambda^{1 / 2} Y^{-1}+\lambda^{-1}\right)\right) .
$$

The result now follows.

Corollary 2.4. There exist positive constants $c_{1}$ and $c_{2}$ such that for $\lambda=\frac{1}{4}+r^{2}$

$$
c_{1} \lambda^{1 / 12} e^{-\pi \lambda^{1 / 2} / 2} \leq \max _{y}\left|y^{1 / 2} K_{i r}(y)\right| \leq c_{2} \lambda^{1 / 12} e^{-\pi \lambda^{1 / 2} / 2} .
$$

Proof. Given the above Lemma [2.3] it is enough to estimate $\mathcal{W}(Y)$. First we consider the lower bound. For $Y=1, \zeta(Y)$ is zero, $\left(\zeta(Y) /\left(1-Y^{-2}\right)\right)^{1 / 4}=2^{-1 / 6}$ and $A i\left(\lambda^{1 / 3} \zeta(1)\right)=A i(0)$ is nonzero; it follows that $M S(1)=2^{-1 / 6} A i(0)+O\left(\lambda^{-2 / 3}\right)$ and that $M E\left(\lambda^{1 / 3} \zeta(1)\right)$ is independent of $\lambda$. Thus, from Theorem 2.2, for $\lambda$ large $\mathcal{W}(1)=M S(1)+O\left(\lambda^{-2 / 3}\right)$ is bounded below by a positive constant, the desired lower estimate.

We are ready to consider the upper bound. From the asymptotics of $|A i(\mu)|$, the $|M E(\mu)|$ are bounded above by a multiple of $\left(|\mu|^{1 / 4}+1\right)^{-1}$ and $\left|B_{0}(\mu) A i^{\prime}(\mu)\right|$ by a multiple of $\left(|\mu|^{7 / 4}+1\right)^{-1}$. Thus $\left|A i\left(\lambda^{1 / 3} \zeta\right)\right|,\left|\lambda^{-2 / 3} B_{0}(\zeta) A i^{\prime}\left(\lambda^{1 / 3} \zeta\right)\right|$ and $M E\left(\lambda^{1 / 3} \zeta\right)$ are all bounded by a multiple of $\left(\lambda^{1 / 12}|\zeta|^{1 / 4}+1\right)^{-1}$. The majorant is decreasing in $\lambda$; it follows that the model solution $\mathcal{W}(Y)$ is bounded for all $\lambda$ by a fixed multiple of $\left(\zeta /\left(1-Y^{-2}\right)\right)^{1 / 4}\left(\lambda_{0}^{1 / 12}|\zeta|^{1 / 4}+1\right)^{-1}, \lambda_{0}$ fixed, which in turn is a bounded function.

Corollary 2.5. Let $g(\mu)$ be a continuously differentiable function with support a compact subinterval of $(0,1)$. For $|L|$ and $\lambda=\frac{1}{4}+r^{2}$ large,

$$
\begin{aligned}
& \int_{0}^{\infty} g\left(|L| \lambda^{-1 / 2} y\right)\left(K_{i r}(|L| y)\right)^{2} y^{-1} d y \\
& =\pi e^{-\pi \lambda^{1 / 2}} \lambda^{-1 / 2}\left(\int_{0}^{\infty} \frac{g(\mu) d \mu}{\mu\left(1-\mu^{2}\right)^{1 / 2}}+O_{g}\left(\lambda^{-1 / 2}\right)\right) .
\end{aligned}
$$

Proof. The first matter is the support of the integrand. By hypothesis the integrand is compactly supported in $(0,1)$. From Section 2.3 and Lemma 2.3 we have on $\operatorname{supp}(g)$ that

$$
\begin{aligned}
& |L| y\left(K_{i r}(|L| y)\right)^{2}=2 \pi^{2} \lambda^{1 / 6} e^{-\pi \lambda^{1 / 2}}(\mathcal{W}(Y))^{2}\left(1+O\left(\lambda^{-1}\right)\right) \\
= & 2 \pi e^{-\pi \lambda^{1 / 2}}\left(\left(Y^{-2}-1\right)^{-1 / 2} \cos ^{2}\left(\frac{2}{3} \lambda^{1 / 2}|\zeta|^{3 / 2}-\frac{\pi}{4}\right)+O\left(\lambda^{-1 / 2}\right)\right) \\
= & \pi e^{-\pi \lambda^{1 / 2}}\left(Y^{-2}-1\right)^{-1 / 2}\left(1+\cos \left(\frac{4}{3} \lambda^{1 / 2}|\zeta|^{3 / 2}-\frac{\pi}{2}\right)+O\left(\lambda^{-1 / 2}\right)\right)
\end{aligned}
$$

for $Y=|L| \lambda^{-1 / 2} y$ and the remainder constant dependent on the interval. The last expansion is used in evaluating the integral with the cosine term to be integrated by parts. 
2.5. In Section 4 we will use the stationary phase expansion for the integral of an exponential with a cubic phase. We now recall the expansion, GS]. For $u$ a smooth function with compact support, write $\mathcal{F}(u)=\hat{u}(\xi)=\int_{-\infty}^{\infty} u(x) e^{-i x \xi} d x$ for the Fourier transform. From the definition of the Airy function we have that $\mathcal{F}\left(e^{i a x^{3} / 3}\right)=2 \pi a^{-1 / 3} A i\left(-a^{-1 / 3} \xi\right)$.

Proposition 2.6. For $n$ a non-negative integer and $u$ a smooth function with compact support,

$$
\begin{aligned}
\int_{-\infty}^{\infty} e^{i \alpha x^{3}} u(x) d x= & 2 \pi(3 \alpha)^{-1 / 3} \sum_{k=0}^{n} \frac{i^{k}}{(3 \alpha)^{k / 3} k !} A i^{(k)}(0) u^{(k)}(0) \\
& +O_{n}\left(\alpha^{-(n+2) / 3}\left(\|u\|_{L^{1}}+\left\|u^{(n+3)}\right\|_{L^{1}}\right)\right) .
\end{aligned}
$$

Proof. First apply Parseval's identity to find that

$$
\int_{-\infty}^{\infty} e^{i a x^{3} / 3} u(x) d x=a^{-1 / 3} \int_{-\infty}^{\infty} A i\left(-a^{-1 / 3} \xi\right) \hat{u}(\xi) d \xi
$$

Then for $p_{n}$ the $n$th Taylor polynomial of $A i$ we have $\left|A i(\tau)-p_{n}(\tau)\right| \leq c_{n}|\tau|^{n+1}$ for all $\tau$, and thus

$$
\begin{gathered}
a^{-1 / 3} \int_{-\infty}^{\infty} A i\left(-a^{-1 / 3} \xi\right) \hat{u}(\xi) d \xi=a^{-1 / 3} \int_{-\infty}^{\infty} p_{n}\left(-a^{-1 / 3} \xi\right) \hat{u}(\xi) d \xi \\
+O\left(a^{-(n+2) / 3} \int_{-\infty}^{\infty}\left|\xi^{n+1} \hat{u}(\xi)\right| d \xi\right) .
\end{gathered}
$$

The explicit terms are evaluated with $\int_{-\infty}^{\infty}(i \xi)^{k} \hat{u}(\xi) d \xi=2 \pi u^{(k)}(0)$. The remainder is bounded from the inequality $|\hat{u}(\xi)|+\left|\xi^{n+3} \hat{u}(\xi)\right| \leq \int_{-\infty}^{\infty}|u(x)|+\left|u^{(n+3)}(x)\right| d x$.

We are now ready to consider the stationary phase expansion for the integral of the Macdonald-Bessel function.

Proposition 2.7. For $g(\mu)$ a smooth function with support a compact subinterval of $(0, \infty), L$ nonzero and $\lambda=\frac{1}{4}+r^{2}$ large, we have

$$
\begin{array}{rl}
\int_{0}^{\infty} g(y) K_{i r}(|L| y) y^{-1 / 2} & d y \\
& =\pi e^{-\lambda^{1 / 2} / 2}|L|^{-1 / 2} \lambda^{-1 / 4}\left(g\left(|L|^{-1} \lambda^{1 / 2}\right)+O_{g}\left(\lambda^{-3 / 4}\right)\right) .
\end{array}
$$

Furthermore, there exist positive constants $c_{0}$ and $c_{1}$, depending on $g$, such that for $|L| \lambda^{-1 / 2} \geq c_{0}$ the integral is $O_{g}\left(e^{-\lambda^{1 / 2} / 2-c_{1}|L|}\right)$.

Proof. The analysis will focus on the expansion for the integral

$$
\int_{0}^{\infty} g\left(|L|^{-1} \lambda^{1 / 2} Y\right) M S(Y) Y^{-1} d Y
$$

for $M S$ the model solution of Definition 2.1. The considerations will be in terms of three regions for the magnitude of $|L| \lambda^{-1 / 2}$. Theorem 2.2 and Lemma 2.3 will be used to relate (11) to the integral of the Macdonald-Bessel function.

We first consider that $|L| \lambda^{-1 / 2}$ is large; in particular, for $y$ in the support of $g$ we assume that $Y=|L| \lambda^{-1 / 2} y \geq 2$. From the expansion (10) and the preparatory discussion to Theorem 2.2 we have that $M S(Y)$ is $O\left(\lambda^{-1 / 12} e^{-\lambda^{1 / 2} Y}\right)$ for $Y \geq 2$, 
and since $Y \geq c|L| \lambda^{-1 / 2}$ on the support of $g$ we have that the integral (11) is $O_{g}\left(e^{-c|L|}\right)$, a suitable bound. We next consider the situation that $|L| \lambda^{-1 / 2}$ is small; in particular, for $y$ in the support of $g$ we assume that $Y=|L| \lambda^{-1 / 2} y \leq 1 / 2$. For this range we will use the expansion (2.4) for $M S(Y)$. The integral (2.7) now has the expansion

$$
\begin{aligned}
\int_{0}^{\infty} g(y) \lambda^{-1 / 12} \pi^{-1 / 2} & \left(Y^{-2}-1\right)^{-1 / 4} \\
& \times\left(\cos \Theta-\frac{\lambda^{-1 / 2}}{24} \frac{\left(3 Y^{2}+2\right)}{\left(1-Y^{2}\right)^{3 / 2}} \sin \Theta\right) y^{-1} d y+O_{g}\left(\lambda^{-13 / 12}\right)
\end{aligned}
$$

for $\Theta=\left(\frac{2}{3} \lambda^{1 / 2}|\zeta|^{3 / 2}-\frac{\pi}{4}\right)$. We prepare to integrate by parts. From the defining relation for $\zeta$ we have that $\frac{2}{3} \frac{d}{d y}|\zeta|^{3 / 2}=\left(1-Y^{2}\right)^{1 / 2} y^{-1}$; it follows that $\left(\frac{2}{3} \frac{d}{d y}|\zeta|^{3 / 2}\right)^{-1}$ and its $y$-derivatives are bounded on $\operatorname{supp}(g)$ for $Y \leq 1 / 2$. Also from the relation we have that $\cos \Theta=\left(\lambda^{1 / 2}\left(1-Y^{2}\right)^{1 / 2} y^{-1}\right)^{-1} \frac{d}{d y} \sin \Theta$, and a corresponding formula for the sine. We can integrate by parts: twice for the cosine term and once for the sine term. The resulting integral is bounded as $O_{g}\left(\lambda^{-13 / 12}\right)$.

We are ready to consider the stationary phase expansion for (11). Akin to Proposition 2.6 from the Fourier transform $\mathcal{F}\left(A i\left(\lambda^{1 / 3} \zeta\right)\right)=\lambda^{-1 / 3} e^{i \lambda^{-1} \xi^{3} / 3}$ we find the expansion $\int_{-\infty}^{\infty} A i\left(\lambda^{1 / 3} \zeta\right) u(\zeta) d \zeta=\lambda^{-1 / 3} u(0)+O\left(\lambda^{-4 / 3}\left(\|u\|_{L^{1}}+\left\|u^{(5)}\right\|_{L^{1}}\right)\right)$. Now since $\frac{d Y}{d \zeta}=\left(\zeta /\left(1-Y^{-2}\right)\right)^{1 / 2}$, the integral (11) after an integration by parts has the above form with $u(\zeta)=g(y)\left(\frac{d Y}{d \zeta}\right)^{3 / 2} Y^{-1}-\lambda^{-1} \frac{d}{d \zeta}\left(g(y)\left(\frac{d Y}{d \zeta}\right)^{3 / 2} Y^{-1} B_{0}(\zeta)\right)$. We are ready to consider the parameter ranges. For $1 / 2 \leq Y \leq 2$ and $y$ in the support of $g$ the quantity $|L| \lambda^{-1 / 2}$ is bounded above and below by positive constants. It follows that $u(\zeta)$ and its $\zeta$-derivatives are uniformly bounded on the specified ranges. We now combine the stationary phase expansion with the earlier considerations to find for $L$ nonzero and $\lambda$ large that $\int_{0}^{\infty} g(y) M S(Y) y^{-1} d y=2^{-1 / 2} \lambda^{-1 / 3} g\left(|L|^{-1} \lambda^{1 / 2}\right)+$ $O_{g}\left(\lambda^{-13 / 12}\right)$. It only remains to relate (2.7) to the integral of the Macdonald-Bessel function. We have from Theorem 2.2 and the accompanying discussion that

$$
\mathcal{W}(Y)=M S(Y)+O\left(\lambda^{-1}\left(1+\lambda^{1 / 12}|\zeta|^{1 / 4}\right)^{-1}\right),
$$

and from Lemma 2.3 the relation to the Macdonald-Bessel function. The desired expansion is now established.

\section{Microlocal Behavior of the Macdonald-Bessel phase}

3.1. We consider elementary (unit-translation invariant, small at infinity) eigenfunctions for the hyperbolic Laplacian, particularly the geometry of their associated phase functions.

Definition 3.1. For $\lambda=\frac{1}{4}+r^{2}>1 / 4$, the Macdonald-Bessel function $K_{i r}$, $m$ a nonzero integer and $z=x+i y, y>0$, let $s=2 \pi m \lambda^{-1 / 2}$ and $\mathcal{K}_{s}(z)=$ $e^{\pi \lambda^{1 / 2} / 2}(2 \pi|m| y)^{1 / 2} K_{i r}(2 \pi|m| y) e^{2 \pi i m x}$.

$\mathcal{K}_{s}$ satisfies $D \mathcal{K}_{s}=-\lambda \mathcal{K}_{s}$ for $D=y^{2}\left(\frac{\partial^{2}}{\partial x^{2}}+\frac{\partial^{2}}{\partial y^{2}}\right)$, and $\mathcal{K}_{s}$ is $O\left(e^{-2 \pi|m| y}\right)$ for $\lambda$ fixed and $y$ large. From Lemma 2.3 and (6) the phase for $\mathcal{K}_{s}$ (below the turning line) is $\Psi=\epsilon F(|s| \operatorname{Im} z)+s R e z$ for $|s| \operatorname{Im} z \leq 1$, where $F(Y)=\int_{Y}^{1}\left(\tau^{-2}-1\right)^{1 / 2} d \tau$. The phase velocity is the hyperbolic gradient of $\Psi, \nabla \Psi=(\operatorname{Imz})^{2}\left(\frac{\partial \Psi}{\partial x}, \frac{\partial \Psi}{\partial y}\right)=$ 


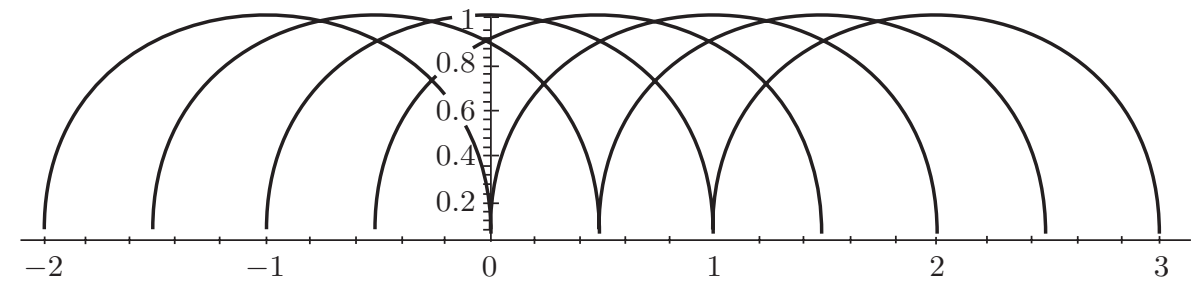

Figure 2. The pencil of geodesics $\mathcal{G}$

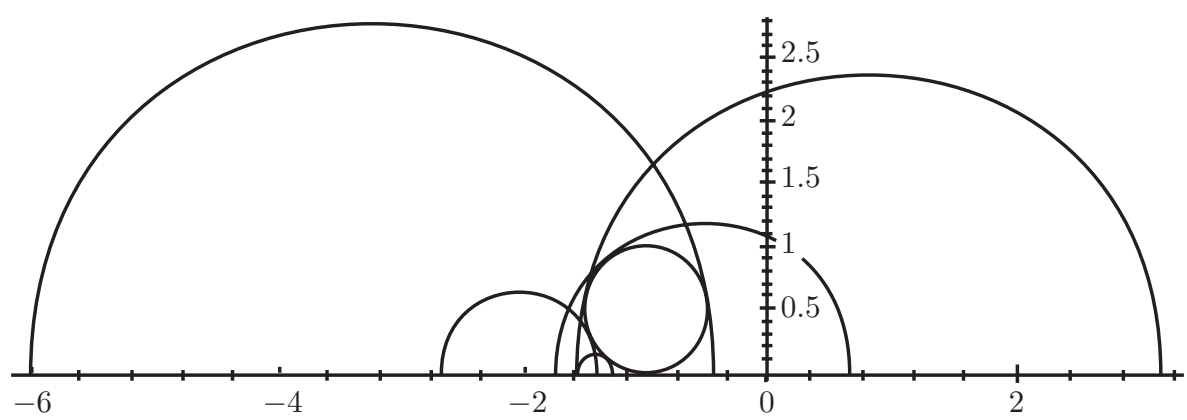

Figure 3. The pencil of geodesics tangent to a horocycle

$\operatorname{Im} z\left(\operatorname{sim} z,-\epsilon\left(1-(\operatorname{sim} z)^{2}\right)^{1 / 2}\right)$, a double-valued vector field on $\{|s| \operatorname{Im} z \leq 1\}$, the exterior of a horocycle. The gradient $\nabla \Psi$ has unit-length and a simple geometric description. Let $\mathcal{G}$ be the pencil of geodesics tangent to the horocycle $\{|s| \operatorname{Im} z=1\}$. The double-field $\nabla \Psi, s>0, \epsilon= \pm 1$, is the rightward pointing unit tangent doublefield to $\mathcal{G}$ (see Figure 2).

That $\nabla \Psi$ has a simple geometric description that illustrates the connection between hyperbolic geometry and eigenfunctions of the hyperbolic Laplacian. The present $F$ arose from the Liouville-Green approximation (see the discussion of the Ricatti equation in Ov, Chapter 6, Section 1.4, pg. 104], and of the eikonal equation in [GS, Exercise 12.1, pg. 140]). The field $\nabla \Psi$ is intrinsically defined; for $A \in S L(2 ; \mathbb{R})$ the phase velocity of $\mathcal{K}_{s} \mid A$ is $\left(A^{-1}\right)_{*} \nabla \Psi$, the tangent double-field to $A^{-1} \mathcal{G}$ (see Figure 3 ). The phase $\Psi$ also gives a (singular) two-sheeted Lagrangian in the cotangent bundle of the upper half plane. In particular, the graph of the differential, $z \in H \rightarrow d \Psi_{\epsilon}=s d x-\epsilon|s|\left((\operatorname{sim} z)^{-2}-1\right)^{1 / 2} d y$, defined for $|s| \operatorname{Im} z \leq 1$, has two distinct sheets, $\epsilon= \pm 1$, for $|s| \operatorname{Im} z<1$ which coincide (fold) for $|s| \operatorname{Im} z=1$.

3.2. Our purpose is to analyze the phase for the product $\mathcal{K}_{s}(A(w)) e^{2 \pi i n R e w}$, $A \in S L(2 ; \mathbb{R}), w \in H, s=2 \pi m \lambda^{-1 / 2}, m, n \in \mathbb{Z}-\{0\}$. For $t=2 \pi n \lambda^{-1 / 2}$ the phase is $\Phi=\epsilon_{*} F(|s| \operatorname{Im} A(w))+\operatorname{Re}(s A(w)+t w)$, which can be written as $\Phi=$ $\epsilon_{*} F(\operatorname{Im} B(u))+\operatorname{Re}(\operatorname{sgn}(s) B(u)+\operatorname{sgn}(t) u)$ for $u=|t| w, v=|s| z, z=A(w)$ and 
$v=B(u), B \in S L(2 ; \mathbb{R})$. The horizontal derivative of the phase is given by

$$
\begin{aligned}
\phi & =2|t|^{-1} \operatorname{Re} \Phi_{w} \\
& =2 \operatorname{Re} \Phi_{u}=\operatorname{sgn}(t)\left(-\epsilon_{2}\left((\operatorname{Im} B(u))^{-2}-1\right)^{1 / 2} \operatorname{Im}^{\prime}(u)-\epsilon_{1} \operatorname{Re} B^{\prime}(u)+1\right)
\end{aligned}
$$

for $\epsilon_{2}=\operatorname{sgn}\left(\epsilon_{*} t\right), \epsilon_{1}=\operatorname{sgn}(-s t)$ and $\operatorname{Im} B(u) \leq 1$. The phase derivative $\phi(u, B)$, $u \in H, B \in S L(2 ; \mathbb{R})$, is an algebraic function. We will give a detailed analysis of its behavior on the height-one horocycle.

We now simplify the phase by introducing a second substitution. All our considerations will be for the horocycle $\operatorname{Im} u=1$. For $\operatorname{Imu}=1$ we have $\operatorname{Im} B(u)=\left|B^{\prime}(u)\right|$. Set $B^{\prime}(u)=\rho e^{-2 i \theta}, 0 \leq \theta<\pi$ and $\tau=\gamma^{2}$ for

$$
B=\left(\begin{array}{ll}
* & * \\
\gamma & \delta
\end{array}\right)=\left(\begin{array}{ll}
* & * \\
c|s t|^{-1 / 2} & d\left|s^{-1} t\right|^{1 / 2}
\end{array}\right) .
$$

We will further specialize to only consider $c>0$ and the quadrant $\operatorname{Re}(c w+d)>0$, in which case $\rho^{-1 / 2} e^{i \theta}=(\gamma u+\delta)=\left|t s^{-1}\right|^{1 / 2}(c w+d), 0<\theta<\pi / 2$. On considering the imaginary part of $\gamma u+\delta=\rho^{-1 / 2} e^{i \theta}$ we find the relation $\gamma=\rho^{-1 / 2} \sin \theta$, or equivalently $\rho \tau=\sin ^{2} \theta$. We now have the relations

$$
\left|t s^{-1}\right|^{1 / 2}(c w+d)=\rho^{-1 / 2} e^{i \theta}, \quad \rho \tau=\sin ^{2} \theta,
$$

$$
\operatorname{Re} B^{\prime}(u)=\rho(1-2 \rho \tau) \quad \text { and } \quad \operatorname{Im} B^{\prime}(u)=-2 \rho(\rho \tau(1-\rho \tau))^{1 / 2} .
$$

From the first two relations and an implicit differentiation we have the further relations

$$
x=R e w=|t|^{-1}\left((\rho \tau)^{-1}-1\right)^{1 / 2}-d c^{-1}
$$

and

$$
\frac{d \rho}{d x}=-2 \rho|t|(\rho \tau(1-\rho \tau))^{1 / 2} .
$$

If $A=\left(\begin{array}{ll}a & b \\ c & d\end{array}\right)$, then $\operatorname{Re} A(w)=a c^{-1}-\operatorname{Re}(c(c w+d))^{-1}$, and since $(c w+d)=$ $\left|s t^{-1}\right|^{1 / 2} \rho^{-1 / 2} e^{i \theta}$ we find that $\operatorname{Re}|s| A(w)=|s| a c^{-1}-\rho\left((\rho \tau)^{-1}-1\right)^{1 / 2}$. We use the above relations to write the phase in terms of the parameters:

$$
\Phi=\epsilon_{*} F(\rho)+\left((\rho \tau)^{-1}-1\right)^{1 / 2}(\operatorname{sgn}(t)-\operatorname{sgn}(s) \rho)+(s a-t d) c^{-1} .
$$

We can also write the phase derivative in terms of the same parameters:

$$
q=\operatorname{sgn}(t) \phi=1-\epsilon_{1} \rho(1-2 \rho \tau)+2 \epsilon_{2}\left(\left(1-\rho^{2}\right) \rho \tau(1-\rho \tau)\right)^{1 / 2}
$$

on the domain $\mathcal{D}=\{(\tau, \rho) \mid \tau \geq 0,0 \leq \rho \leq 1, \rho \tau \leq 1\}$. We further find for the quantities

$$
\mathcal{A}=\left(\left(1+\epsilon_{1} \rho\right) \rho \tau\right)^{1 / 2} \text { and } \mathcal{B}=\left((1-\rho \tau)\left(1-\epsilon_{1} \rho\right)\right)^{1 / 2}
$$

that

$$
q=\left(\mathcal{A}+\epsilon_{2} \mathcal{B}\right)^{2} .
$$

Proposition 3.2. Notation as above. The phase derivative $q$ has domain $\mathcal{D}$ and the factorization $q=\left(\mathcal{A}+\epsilon_{2} \mathcal{B}\right)^{2}$. The phase derivative vanishes with multiplicity two for $\epsilon_{2}=-1, \rho=S_{\epsilon_{1}}(\tau)=\left(2 \tau+\epsilon_{1}\right)^{-1}$, and is otherwise nonzero.

Proof. The conclusions follow from the factorization of $q$ and the observation that the equation $\mathcal{A}^{2}=\mathcal{B}^{2}$ reduces to $\rho=\left(2 \tau+\epsilon_{1}\right)^{-1}$. 
3.3. We now consider the behavior of the phase on the stationary locus. We start be defining the integral

$$
Q=-\int_{S_{\epsilon_{1}}(\tau)}^{\kappa}\left(\mathcal{A}+\epsilon_{2} \mathcal{B}\right)^{2}\left(2 \rho|t|(\rho \tau(1-\rho \tau))^{1 / 2}\right)^{-1} d \rho .
$$

Observe, for $x=$ Rew, from (13) and the definition of $\phi$ that $\frac{d Q}{d x}=q\left(\frac{d \kappa}{d x}\right)^{-1}\left(\frac{d \kappa}{d x}\right)=$ $\operatorname{sgn}(t) \phi=t^{-1} \frac{d \Phi}{d x}$. We next define the quantity (note that $\tau=c^{2}|s t|^{-1}$ )

$$
\begin{gathered}
Q_{0}(t, s)=2\left(1+\tau^{-1}\right)^{1 / 2}-\log \left(2 \tau+1+2\left(\tau^{2}+\tau\right)^{1 / 2}\right) \\
-(|s| a+t d) c^{-1}
\end{gathered}
$$

Proposition 3.3. Notation as above. For $\epsilon_{1}=-\epsilon_{2}=1, t>0$, the phase is given as $\Phi=t Q+Q_{0}$. The quantity $t Q$ has an analytic cube-root $\Xi=(t Q)^{1 / 3}$ which vanishes to first-order precisely on $S_{1}(\tau)$. For $\rho$ depending on $x=$ Rew the cube-root $\Xi$ is an invertible analytic function of $x$.

Proof. By definition $Q$ vanishes on $S_{1}(\tau)$, and $\Phi_{x}=(t Q)_{x}$. It follows that $\Phi=$ $t Q+\left.\Phi\right|_{S_{1}}$. To evaluate $\left.\Phi\right|_{S_{1}}$ combine formula (14) for $\Phi$, formula (6) for $F$, and then evaluate on $S_{1}$ by substituting $\rho=(2 \tau+1)^{-1}$; the result is $\left.\Phi\right|_{S_{1}}=Q_{0}$. Next observe that the integrand for $Q$ is nonnegative, and vanishes to second-order on $S_{1}$. It follows that $Q$ vanishes to third-order on $S_{1}$ and is otherwise nonzero. It further follows that $\Xi=(t Q)^{1 / 3}$ is analytic, vanishing to first-order on $S_{1}$. We consider the dependence on $x$. From (13) the derivative $\frac{d \rho}{d x}$ is negative; it follows that $\Xi_{x}$ is positive on $S_{1}$. On the complement of $S_{1}$ we have $(t Q)_{x}=t q>0$, and thus $\Xi_{x}$ is positive. It now follows that $\Xi$ is an invertible function of $x$.

\section{THE STATIONARY PHASE EXPANSION AND THE FOURIER COEFFICIENT RELATION}

4.1. We consider the stationary phase expansion of the integral of the product of a Macdonald-Bessel function, exponentials and a test function

$$
\mathcal{K}_{s}(A(w)) e^{2 \pi i n \operatorname{Rew}} \tilde{h}(w), \quad A \in S L(2 ; \mathbf{R}),
$$

over the horocycle $\left\{2 \pi|n| \lambda^{-1 / 2}\right.$ Imw $\left.=1\right\}$. We start in Section 4.2 by setting the parameter ranges for the integration. The support of the test function $\tilde{h}$ is specified to avoid the special singular point $(\tau, \rho)=(0,1)$ and to ensure that the support is transverse to the stationary locus $S_{\epsilon_{1}}(\tau)$ given in Proposition 3.2, By introducing a partition of unity we can separately consider the integrals for the different regions of the Airy function. We start in Section 4.3. Proposition 4.2. with the region of exponential decay. In Section 4.4 we consider the turning region. We first use the Airy differential equation and integration by parts to obtain a recursion among integrals of the Airy function. Then in Propositions 4.4 and 4.5 we use the recursion to obtain the desired estimate. In Section 4.5, Proposition 4.6 we compute the contribution to the integral from a neighborhood of the stationary locus, the stationary phase contribution. In Section 4.6. Proposition 4.7 we estimate the contribution from the tail. In Section 4.7 Theorem 4.8 we combine all our considerations and standard bounds to derive the fundamental relation for the Fourier coefficients of an automorphic eigenfunction. 
4.2. We will now set the parameters for the analysis of the integrals. Given $0<t_{0}<t_{1}$, choose $\beta>4$ and define $\alpha$ so that $(\alpha-1)^{1 / 2}=2 t_{1}+(\beta-1)^{1 / 2}$. Let $h(x)$ be a smooth function on $\mathbf{R}$ with support contained in $(0,2)$. Recall the setup of the previous section, and start with $A=\left(\begin{array}{ll}a & b \\ c & d\end{array}\right) \in S L(2 ; \mathbf{R}), c>$ $0,(A(\infty)$ finite $), s \in \mathbf{R}-\{0\}, t_{0} \leq t \leq t_{1}$, and $w \in H$ with $t$ Imw $=1$. Recall the associated parameters $\tau=c^{2}|s t|^{-1}, \rho e^{-2 i \theta}=\left|s t^{-1}\right|(c w+d)^{-2}($ with $\operatorname{Re}(c w+d)>0$ and $0<\theta<\pi / 2)$ and the basic relation $\rho \tau=\sin ^{2} \theta$. Introduce the shifted function $\tilde{h}(w)=h\left(\right.$ Rew $\left.-(\beta-1)^{1 / 2} \operatorname{Im} w+d c^{-1}\right)$.

Proposition 4.1. Notation as above. For $w \in H$ with $t_{1}^{-1} \leq I m w \leq t_{0}^{-1}$ on the support of $\tilde{h}(w)$ the associated parameters $\rho, \tau$, s satisfy $\alpha^{-1}<\rho \tau<\beta^{-1}$ and $c_{1}|s| \leq \rho \leq c_{2}|s|$ for positive constants $c_{1}, c_{2}$.

Proof. By the specification on the support of $h$, for $w \in \operatorname{supp}(\tilde{h})$ we have $0<$ Rew $-(\beta-1)^{1 / 2} \operatorname{Imw}+d c^{-1}<2$. This inequality can be rewritten as

$$
(\beta-1)^{1 / 2}<\operatorname{Re}\left(w+d c^{-1}\right)(\operatorname{Im} w)^{-1}<(\beta-1)^{1 / 2}+2(\operatorname{Im} w)^{-1} \leq(\alpha-1)^{1 / 2}
$$

with the last inequality from the restriction on $\alpha, \beta$ for $t_{1}^{-1} \leq \operatorname{Imw} \leq t_{0}^{-1}$. Now $\operatorname{Re}\left(w+d c^{-1}\right)(I m w)^{-1}=\cot \theta$ for $\theta=\arg (c w+d)$, and so we have $(\beta-1)^{1 / 2}<$ $\cot \theta<(\alpha-1)^{1 / 2}$, which in turn is equivalent to $\alpha^{-1}<\sin ^{2} \theta<\beta^{-1}$. The first conclusion now follows from the relation $\rho \tau=\sin ^{2} \theta$. Since $w$ and $t$ are restricted to compact sets, the second conclusion follows from the relation $\rho=\left|s t^{-1} A^{\prime}(w)\right|$.

Now we specify a partition of unity for the positive half-line. Select four smooth non-negative functions $g_{0}, g_{1}, g_{2}$ and $g_{3}$ satisfying $\sum_{j=0}^{3} g_{j}(\rho) \equiv 1$ for $0 \leq \rho<\infty$, with $g_{0}$ having support in $(1, \infty], g_{1}$ having support in a neighborhood of unity (to be further specified in Proposition 4.5), $g_{2}$ having compact support in $(0,1)$ with $g_{2}$ identically unity on a neighborhood of $\left[1-2 \beta^{-1}, 1-2 \alpha^{-1}\right]$, and finally $g_{3}$ having support in $\left[0, \beta(2 \alpha)^{-1}\right)$. We will henceforth write $\|u\|_{H^{k}}$ for the Sobolev $k$-norm of a (smooth) function $u$ (the sum of the $L^{2}$-norms of the first $k$ derivatives).

We now introduce the principal integrals of interest. For $A \in S L(2 ; \mathbf{R}), A(\infty)$ finite, $t=2 \pi n \lambda^{-1 / 2}>0, s=2 \pi m \lambda^{-1 / 2}, m, n \in \mathbf{Z}-\{0\}, \rho=\left|s t^{-1}\right|\left|A^{\prime}(w)\right|, j=$ $0,1,2,3$, set

$$
\mathcal{J}_{j}(t, s)=\int_{t I m w=1} \mathcal{K}_{s}(A(w)) e^{2 \pi i n R e w} \tilde{h}(w) g_{j}(\rho) d \operatorname{Rew}
$$

and

$$
\begin{gathered}
\mathcal{I}_{j}(t, s)=2^{1 / 2} \pi \lambda^{1 / 12} \int_{t \operatorname{Imw} w=1}\left(\zeta(\rho) /\left(1-\rho^{-2}\right)\right)^{1 / 4}\left(\operatorname{Ai}\left(\lambda^{1 / 3} \zeta(\rho)\right)\right. \\
\left.+\lambda^{-2 / 3} B_{0}(\zeta(Y)) A i^{\prime}\left(\lambda^{1 / 3} \zeta(Y)\right)\right) e^{2 \pi i m \operatorname{Re} A(w)+2 \pi i n \operatorname{Rew}} \tilde{h}(w) g_{j}(\rho) d \operatorname{Rew}
\end{gathered}
$$

(see Section 2.3 and Definition 3.1] also note that for $\operatorname{tm} w=1$ that $Y=|s| \operatorname{Im} A(w)$ $\left.=\left|s t^{-1}\right|\left|A^{\prime}(w)\right|=\rho\right)$. From Proposition $4.1 \rho$ is comparable to $|s|$, and thus the above integrals are for the different $|s|$ ranges (for example $g_{0}(\rho), \mathcal{J}_{0}, \mathcal{I}_{0}$ are identically zero if $|s|$ is sufficiently small). 
4.3. We first estimate the integral in the region of $\rho$ large.

Proposition 4.2. Notation as above. There exist positive constants $\hat{c}$ and $\hat{\hat{c}}$ such that, for $t_{0} \leq t \leq t_{1}, s=2 \pi m \lambda^{-1 / 2}$, and $\lambda$ sufficiently large,

$$
\left|\mathcal{J}_{0}(t, s)\right| \leq \hat{\hat{c}}\|h\|_{H^{0}}|m|^{-1} e^{-\hat{c}|m|} .
$$

Proof. The first matter is to bound $\mathcal{K}_{s}(A(w))$. From Section 2.2 the function $\frac{2}{3}(\zeta(\rho))^{3 / 2}$ is increasing, positive for $\rho>1$ and satisfies $\frac{2}{3}(\zeta(\rho))^{3 / 2}=\rho-\pi / 2+$ $O\left(\rho^{-1}\right)$ for $\rho$ large. In particular, there exists a positive constant $c_{0}$ such that $\frac{2}{3}(\zeta(\rho))^{3 / 2} \geq c_{0} \rho$ on $\operatorname{supp}\left(g_{0}\right)$. It follows from Theorem 2.2 Lemma 2.3. Definition 3.1 and (9) that $\left|\mathcal{K}_{s}(A(w))\right| \leq c_{1} e^{-\lambda^{1 / 2} c_{0} \rho}$ on $\operatorname{supp}\left(g_{0}\right)$ for a suitable constant $c_{1}$. The next matter is to recall from (13) the dependence of $\rho$ on $x=$ Rew. From Proposition 4.1, on $\operatorname{supp}(\tilde{h})$ the product $\rho \tau$ is bounded by $\alpha^{-1}<\rho \tau<\beta^{-1}$; it follows that $\left|\frac{d \rho}{d x}\right| \geq c_{2} \rho$ on $\operatorname{supp}(\tilde{h})$ for $c_{2}$ a positive constant (since $t_{0} \leq t$ ). We combine the above bounds to find that $\left|\mathcal{J}_{0}\right| \leq c_{3} \int_{1}^{\infty} e^{-\lambda^{1 / 2} c_{0} \rho}|\tilde{h}(w)| g_{0}(\rho) \rho^{-1} d \rho$. From Proposition 4.1 we have that $\rho \geq c^{\prime}|s|$ for a positive constant $c^{\prime}$. The desired estimate for $\mathcal{J}_{0}$ follows.

4.4. We are ready to estimate the integral $\mathcal{J}_{1}$ for the turning region of $\mathcal{K}_{s}$. First we consider technical results for the asymptotics of the Airy-exponential integral. We introduce, for smooth functions $f$ and $g$ (with $\operatorname{supp}(g)$ compact and $\frac{d f}{d \sigma}$ not vanishing on $\operatorname{supp}(g))$ and $\lambda$ positive, the integrals

$$
\mathcal{S}(g)=\int_{-\infty}^{\infty} e^{i \lambda^{1 / 2} f(\sigma)} A i\left(\lambda^{1 / 3} \sigma\right) g(\sigma) d \sigma
$$

and

$$
\mathcal{T}(g)=\int_{-\infty}^{\infty} e^{i \lambda^{1 / 2} f(\sigma)} A i^{\prime}\left(\lambda^{1 / 3} \sigma\right) g(\sigma) d \sigma .
$$

From the coarse estimates $|A i(\mu)| \leq c_{*}|\mu|^{-1 / 4}$ and $\left|A i^{\prime}(\mu)\right| \leq c_{*}(1+|\mu|)^{1 / 4}$, valid for all $\mu$, we immediately have that $\mathcal{S}(g)$ is $O_{c_{0}}\left(\lambda^{-1 / 12}\|g\|_{H^{0}}\right)$ and $\mathcal{T}(g)$ is $O_{c_{0}}\left(\lambda^{1 / 12}\|g\|_{H^{0}}\right)$ for $\lambda$ large and $\operatorname{supp}(g) \subset\left[-c_{0}, c_{0}\right]$. There are actually relations for $\mathcal{S}$ and $\mathcal{T}$ from integrating by parts. The relations involve the multiplication operators $S$ for multiplying by $\sigma, M$ for multiplying by $\left(\frac{d f}{d \sigma}\right)^{-1}$, and the differentiation operator $D=\frac{d}{d \sigma}$. We integrate by parts with $d u=e^{i \lambda^{1 / 2} f(\sigma)} \frac{d f}{d \sigma} d \sigma ;$ for $\mathcal{S}$ take $v=A i\left(\lambda^{1 / 3} \sigma\right)\left(\frac{d f}{d \sigma}\right)^{-1} g(\sigma)$ and for $\mathcal{T}$ take $v=A i^{\prime}\left(\lambda^{1 / 3} \sigma\right)\left(\frac{d f}{d \sigma}\right)^{-1} g(\sigma)$. The resulting relations are

$$
\mathcal{S}(g)=i \lambda^{-1 / 2} \mathcal{S}(D M g)+i \lambda^{-1 / 6} \mathcal{T}(M g)
$$

and

$$
\mathcal{T}(g)=i \lambda^{1 / 6} \mathcal{S}(S M g)+i \lambda^{-1 / 2} \mathcal{T}(D M g) .
$$

Proposition 4.3. Notation as above. There is a first-order differential operator $T$ such that

$$
\mathcal{S}(g)=\mathcal{S}\left(\left[S^{4} M^{8}+\lambda^{-1 / 2} S^{3} T\right] g\right)+O\left(\lambda^{-13 / 12}\|g\|_{H^{5}}\right)
$$

and

$$
\mathcal{T}(g)=i \lambda^{1 / 6} \mathcal{S}(S M g)+O\left(\lambda^{-5 / 12}\|g\|_{H^{2}}\right)
$$

with the remainder constants being bounded in terms of $c_{0},\left\|\left(\frac{d f}{d \sigma}\right)^{-1}\right\|_{C^{0}\left(\left[-c_{0}, c_{0}\right]\right)}$ and $\|f\|_{C^{5}\left(\left[-c_{0}, c_{0}\right]\right)}$. 
Proof. Start with the first relation and twice substitute in the second relation for the resulting $\mathcal{T}$-terms. The result is the relation $\mathcal{S}(g)=\mathcal{S}\left(\left[-S M^{2}+\lambda^{-1 / 2} P\right] g\right)-$ $i \lambda^{-7 / 6} \mathcal{T}\left(D M D M^{2} g\right)$ for $P=i D M-i S M D M^{2}$, a first order differential operator. The $\mathcal{T}$-term is immediately $O_{c_{0}}\left(\lambda^{-13 / 12}\|g\|_{H^{2}}\right)$. Now we use this relation to substitute three times in succession for the $\mathcal{S}$-terms on the right hand side. The result is $\mathcal{S}(g)=\mathcal{S}\left(\left[-S M^{2}+\lambda^{-1 / 2} P\right]^{4} g\right)+O_{c_{0}}\left(\lambda^{-13 / 12}\|g\|_{H^{5}}\right)$. Now expand the product to find $\left[-S M^{2}+\lambda^{-1 / 2} P\right]^{4}=S^{4} M^{8}+\lambda^{-1 / 2} S^{3} T_{1}+\lambda^{-1} S^{2} T_{2}+\lambda^{-3 / 2} S T_{3}+\lambda^{-2} T_{4}$ for $T_{j}$ an order- $j$ differential operator. The desired expansion for $\mathcal{S}$ now follows on applying the general bound for the operator $\mathcal{S}$. We next consider the basic relation for $\mathcal{T}$ and substitute in for the $\mathcal{T}$-term on the right hand side. The result is $\mathcal{T}(g)=\mathcal{S}\left(\left[i \lambda^{1 / 6} S M-\lambda^{-1 / 3} S M D M\right] g\right)-\lambda^{-1} \mathcal{T}(D M D M g)$. On applying the general bounds for $\mathcal{S}$ and $\mathcal{T}$ we obtain the desired expansion for $\mathcal{T}$.

Proposition 4.4. Let $f(\sigma), g(\sigma)$ be smooth functions with $\operatorname{supp}(g) \subset\left[-c_{0}, c_{0}\right]$. If $f(\sigma)$ and $f(\sigma) \pm \frac{2}{3}|\sigma|^{3 / 2}$ are not stationary on $\operatorname{supp}(g)$, then $\mathcal{S}(g)$ is $O\left(\lambda^{-13 / 12}\|g\|_{H^{5}}\right)$ and $\mathcal{T}(g)$ is $O\left(\lambda^{-5 / 12}\|g\|_{H^{5}}\right)$, where the remainder constants are bounded in terms of $c_{0},\left\|\left(\frac{d f}{d \sigma}\right)^{-1}\right\|_{C^{0}\left(\left[-c_{0}, c_{0}\right]\right)},\left\|\left(\frac{d f}{d \sigma} \pm|\sigma|^{1 / 2}\right)^{-1}\right\|_{C^{0}\left(\left[-c_{0}, c_{0}\right]\right)}$ and $\|f\|_{C^{5}\left(\left[-c_{0}, c_{0}\right]\right)}$.

Proof. The main step is to estimate the integrals $\mathcal{S}\left(S^{3} g\right)$ and $\mathcal{T}(S g)$. We will consider the integrals $\mathcal{S}_{+}, \mathcal{T}_{+}$for $\sigma$ positive and $\mathcal{S}_{-}, \mathcal{T}_{-}$for $\sigma$ negative separately. For $\mu$ positive we have $A i(\mu)=\left(2 \pi^{1 / 2} \mu^{1 / 4}\right)^{-1} e^{-\frac{2}{3} \mu^{3 / 2}}\left(1-\frac{5}{48} \mu^{-3 / 2}+O\left(\mu^{-3}\right)\right)$ and $A i^{\prime}(\mu)=\left(2 \pi^{1 / 2}\right)^{-1} \mu^{1 / 4} e^{-\frac{2}{3} \mu^{3 / 2}}\left(1+O\left(\mu^{-3 / 2}\right)\right)$, see Section [2.4 and Ov, Chap 11, Sec. 1]; both expansions are valid for all positive $\mu$ with a fixed remainder constant. We use the expansions to evaluate $\mathcal{S}_{+}\left(S^{3} g\right)$ and $\mathcal{T}_{+}(S g)$. The remainder term associated to $\mathcal{S}_{+}\left(S^{3} g\right)$ is

$$
O\left(\lambda^{-13 / 12} \int_{0}^{\infty} e^{-\frac{2}{3} \lambda^{1 / 2} \sigma^{3 / 2}} \sigma^{-1 / 4}|g(\sigma)| d \sigma\right)
$$

which is $O_{c_{0}}\left(\lambda^{-13 / 12}\|g\|_{H^{0}}\right)$. The remainder term associated to $\mathcal{T}_{+}(S g)$ is

$$
O\left(\lambda^{-5 / 12} \int_{0}^{\infty} e^{-\frac{2}{3} \lambda^{1 / 2} \sigma^{3 / 2}} \sigma^{-1 / 4}|g(\sigma)| d \sigma\right),
$$

which is $O_{c_{0}}\left(\lambda^{-5 / 12}\|g\|_{H^{0}}\right)$. The explicit integrals respectively are

$$
\begin{array}{ll}
\lambda^{-1 / 12} & \int_{0}^{\infty} e^{\lambda^{1 / 2}\left(i f(\sigma)-\frac{2}{3} \sigma^{3 / 2}\right)} \sigma^{11 / 4} g(\sigma) d \sigma, \\
\lambda^{-7 / 12} & \int_{0}^{\infty} e^{\lambda^{1 / 2}\left(i f(\sigma)-\frac{2}{3} \sigma^{3 / 2}\right)} \sigma^{5 / 4} g(\sigma) d \sigma
\end{array}
$$

and

$$
\lambda^{1 / 12} \int_{0}^{\infty} e^{\lambda^{1 / 2}\left(i f(\sigma)-\frac{2}{3} \sigma^{3 / 2}\right)} \sigma^{5 / 4} g(\sigma) d \sigma
$$

We integrate by parts with $d u=e^{\lambda^{1 / 2}\left(i f(\sigma)-\frac{2}{3} \sigma^{3 / 2}\right)}\left(i \frac{d f}{d \sigma}-\sigma^{1 / 2}\right) d \sigma$, respectively twice, once and once (the factors of $\sigma$ and the compact support of $g$ provide that the boundary terms vanish). In consequence we find that $\mathcal{S}_{+}\left(S^{3} g\right)$ is $O_{c_{0}}\left(\lambda^{-13 / 12}\|g\|_{H^{2}}\right)$ and $\mathcal{T}_{+}(S g)$ is $O_{c_{0}}\left(\lambda^{-5 / 12}\|g\|_{H^{1}}\right)$.

For $\mu$ positive, recall from Section 2.4 that

$$
A i(-\mu)=\left(\pi^{1 / 2} \mu^{1 / 4}\right)^{-1}\left(\cos \left(\frac{2}{3} \mu^{3 / 2}-\frac{\pi}{4}\right)+\frac{5}{48} \mu^{-3 / 2} \sin \left(\frac{2}{3} \mu^{3 / 2}-\frac{\pi}{4}\right)+O\left(\mu^{-3}\right)\right)
$$


and

$$
A i^{\prime}(-\mu)=\pi^{-1 / 2} \mu^{1 / 4}\left(\sin \left(\frac{2}{3} \mu^{3 / 2}-\frac{\pi}{4}\right)+O\left(\mu^{-3 / 2}\right)\right),
$$

Ov, Chap. 11, Sec. 1]; both expansions are valid for all positive $\mu$ with a fixed remainder constant. We use the expansions to evaluate $\mathcal{S}_{-}\left(S^{3} g\right)$ and $\mathcal{T}_{-}(S g)$. The remainder associated to $\mathcal{S}_{-}\left(S^{3} g\right)$ is

$$
O\left(\lambda^{-13 / 12} \int_{-\infty}^{0}(-\sigma)^{-1 / 4}|g(\sigma)| d \sigma\right),
$$

which is $O_{c_{0}}\left(\lambda^{-13 / 12}\|g\|_{H^{0}}\right)$; the remainder associated to $\mathcal{T}_{-}(S g)$ is

$$
O\left(\lambda^{-5 / 12} \int_{-\infty}^{0}(-\sigma)^{-1 / 4}|g(\sigma)| d \sigma\right)
$$

which is $O_{c_{0}}\left(\lambda^{-5 / 12}\|g\|_{H^{0}}\right)$. The explicit integrals respectively are

$$
\begin{array}{ll}
\lambda^{-1 / 12} & \int_{-\infty}^{0} e^{i \lambda^{1 / 2} f(\sigma)} \cos \left(\frac{2}{3} \lambda^{1 / 2}(-\sigma)^{3 / 2}-\frac{\pi}{4}\right)(-\sigma)^{11 / 4} g(\sigma) d \sigma, \\
\lambda^{-7 / 12} & \int_{-\infty}^{0} e^{i \lambda^{1 / 2} f(\sigma)} \sin \left(\frac{2}{3} \lambda^{1 / 2}(-\sigma)^{3 / 2}-\frac{\pi}{4}\right)(-\sigma)^{5 / 4} g(\sigma) d \sigma
\end{array}
$$

and

$$
\lambda^{1 / 12} \int_{-\infty}^{0} e^{i \lambda^{1 / 2} f(\sigma)} \sin \left(\frac{2}{3} \lambda^{1 / 2}(-\sigma)^{3 / 2}-\frac{\pi}{4}\right)(-\sigma)^{5 / 4} g(\sigma) d \sigma .
$$

We express the cosine and sine as a sum of exponentials and integrate by parts with $d u=e^{i \lambda^{1 / 2}\left(f(\sigma) \pm \frac{2}{3}(-\sigma)^{3 / 2}\right)}\left(\frac{d f}{d \sigma} \pm(-\sigma)^{1 / 2}\right) d \sigma$ respectively twice, once and once. Note that by hypothesis $f(\sigma) \pm \frac{2}{3}(-\sigma)^{3 / 2}$ is non-stationary on $\operatorname{supp}(g)$, hence bounded away from zero, and note that the boundary terms vanish given the factors of $\sigma$ and the compact support of $g$. We find that $\mathcal{S}_{-}\left(S^{3} g\right)$ is $O_{c_{0}}\left(\lambda^{-13 / 12}\|g\|_{H^{2}}\right)$ and $\mathcal{T}_{-}(S g)$ is $O_{c_{0}}\left(\lambda^{-5 / 12}\|g\|_{H^{1}}\right)$. On combining the bounds for positive and negative integration regions with the expansions from Proposition 4.3 and the first relation, we find that $\mathcal{S}(g)$ is $O\left(\lambda^{-13 / 12}\|g\|_{H^{5}}\right)$ and $\mathcal{T}(g)$ is $O\left(\lambda^{-5 / 12}\|g\|_{H^{2}}\right)$ with the remainder constants with dependence as claimed.

Proposition 4.5. Notation as above. There exists a neighborhood $\mathcal{U}$ of unity disjoint from $\left[1-2 \beta^{-1}, 1-2 \alpha^{-1}\right]$ such that if $\operatorname{supp}\left(g_{1}\right) \subset \mathcal{U}$ and $t_{0} \leq t \leq t_{1}$, then $\mathcal{J}_{1}(t, x)$ is $O\left(\lambda^{-1}\|h\|_{H^{5}}\right)$ for $\lambda$ large, with the remainder constant depending on $A, \beta, t_{0}$ and $t_{1}$.

Proof. We start by considering the remainder in the approximation of Theorem 2.2 From the coarse estimate $M E(\mu) \leq c_{*}|\mu|^{-1 / 4}$ we find that $\mathcal{W}(\rho)=M S(\rho)+$ $O\left(\lambda^{-13 / 12}\left|1-\rho^{-2}\right|^{-1 / 4}\right)$. From Lemma 2.3 and Definition 3.1 we find that

$$
\mathcal{J}_{1}(t, s)=\mathcal{I}_{1}(t, s)+O\left(\lambda^{-1} \int_{-\infty}^{\infty}\left|1-\rho^{-2}\right|^{-1 / 4} \tilde{h}(w) g_{1}(\rho) d R e w\right) ;
$$

the integral $\int_{-\infty}^{\infty}\left|1-\rho^{-2}\right|^{-1 / 4} \tilde{h}(w) g_{1}(\rho) d R e w$ is in turn bounded in terms of $\|h\|_{L^{2}}$. It remains to bound $\mathcal{I}_{1}(t, s)$.

The quantity $\zeta$ is an invertible analytic function of $\rho$, which in turn is an invertible analytic function of $x=R e w$. Thus the integrand of $\mathcal{I}_{1}$ can alternately be expressed 
as a function of $\zeta$. Specifically, for $\sigma=\zeta(\rho(x)), f=s \operatorname{Re} A(w)+t \operatorname{Rew}, \hat{g}(\sigma)=$ $\left(\zeta(\rho) /\left(1-\rho^{-2}\right)\right)^{1 / 4} \tilde{h}(w) g_{1}(\rho)\left(\frac{d \zeta(\rho(x))}{d x}\right)^{-1}$, and $\hat{\hat{g}}(\sigma)=B_{0}(\sigma) \hat{g}(\sigma)$, we have that

$$
\mathcal{I}_{1}(t, s)=2^{1 / 2} \pi \lambda^{1 / 12} \int_{-\infty}^{\infty} e^{i \lambda^{1 / 2} f(\sigma)}\left(A i\left(\lambda^{1 / 3} \sigma\right) \hat{g}(\sigma)+\lambda^{-2 / 3} A i^{\prime}\left(\lambda^{1 / 3} \sigma\right) \hat{\hat{g}}(\sigma)\right) d \sigma
$$

with $\hat{g}(\sigma), \hat{\hat{g}}(\sigma)$ of compact support and the norms of $\hat{g}, \hat{\hat{g}}$ comparable to the norm of $h$, since $\left(\zeta(\rho) /\left(1-\rho^{-2}\right)\right)^{1 / 4}\left(\frac{d \zeta(\rho(x))}{d x}\right)^{-1}, B_{0}(\zeta)$ and $\zeta(\rho(x))$ are analytic on $\operatorname{supp}\left(g_{1}\right)$, which is compact.

We wish to show that $f(\sigma)$ and $f(\sigma) \pm \frac{2}{3}|\sigma|^{3 / 2}$ are not stationary on $\operatorname{supp}(\hat{g})$ and $\operatorname{supp}(\hat{\hat{g}})$ (in preparation for applying Proposition 4.4). It is equivalent to show that $f=s R e A(w)+t$ Rew and $f \pm \frac{2}{3}|\zeta|^{3 / 2}$ are not stationary in $x=$ Rew on $\operatorname{supp}\left(\tilde{h}(w) g_{1}(\rho)\right)$. Now from Section 3.2 ,

$$
\frac{d f}{d x}=t\left(-\epsilon_{1} \rho(1-2 \tau \rho)+1\right)
$$

and

$$
\frac{d}{d x}\left(f+\epsilon_{*} \frac{2}{3}|\zeta|^{3 / 2}\right)=t\left(1-\epsilon_{1}\left(\rho-2 \tau \rho^{2}\right)+2 \epsilon_{2}\left(\tau\left(\rho-\tau \rho^{2}\right)\left|1-\rho^{2}\right|\right)^{1 / 2}\right)
$$

for $\epsilon_{*}= \pm 1, \epsilon_{1}=\operatorname{sgn}(-s t)$, and $\epsilon_{2}=\operatorname{sgn}\left(\epsilon_{*} t(1-\rho)\right)$. From Proposition 4.1, on $\operatorname{supp}(\tilde{h})$ we have $0<1-2 \beta^{-1}<1-2 \tau \rho<1-2 \alpha^{-1}<1$, and thus $\frac{d f}{d x}, \epsilon_{1}= \pm 1$, is bounded away from zero for $\rho$ sufficiently close to unity (this is a further restriction for $\left.\operatorname{supp}\left(g_{1}\right)\right)$. We likewise have that

$$
\frac{d}{d x}\left(f+\epsilon_{*} \frac{2}{3}|\zeta|^{3 / 2}\right)=t\left(1-\epsilon_{1}\left(\rho-2 \tau \rho^{2}\right)\right)+O\left(\left|\rho^{2}-1\right|^{1 / 2}\right)
$$

with $0<1-2 \tau \rho<1-2 \alpha^{-1}<1 ; \frac{d}{d x}\left(f+\epsilon_{*} \frac{2}{3}|\zeta|^{3 / 2}\right)$ is also bounded away from zero for $\rho$ sufficiently close to unity. In summary, for $\operatorname{supp}\left(g_{1}\right) \subset \mathcal{U}$, a specified neighborhood of unity, the hypotheses of Proposition 4.4 are satisfied for $\mathcal{I}_{1}(t, x)$. The desired estimate for the integral follows.

4.5. We consider the integral in the region where the phase is stationary. We start with definitions of the quantities that appear in the expansion; set

$$
\begin{aligned}
G(\rho, \tau, t)=- & \left(2 \rho|t|(\rho \tau(1-\rho \tau))^{1 / 2}\left(\rho^{-2}-1\right)^{1 / 4} \frac{d \Xi}{d \rho}\right)^{-1} \\
& \times\left(1-\frac{i\left(3 \rho^{2}+2\right)}{24 \lambda^{1 / 2}\left(1-\rho^{2}\right)^{3 / 2}}\right) h\left(|t|^{-1}\left(\left((\rho \tau)^{-1}-1\right)^{1 / 2}-(\beta-1)^{1 / 2}\right)\right)
\end{aligned}
$$

and

$$
G_{k}(\tau, t)=\left.\left(\left(\frac{d \Xi}{d \rho}\right)^{-1} \frac{d}{d \rho}\right)^{k} G(\rho, \tau, t)\right|_{\rho=(2 \tau+1)^{-1}}
$$

for $\Xi^{3}=Q$ with $\epsilon_{1}=-\epsilon_{2}=1$ (see Proposition 3.3).

Proposition 4.6. Notation as above. For $t_{0} \leq t \leq t_{1}, s<0$, and $\lambda$ large, $\mathcal{J}_{2}(t, s)=2^{1 / 2} \pi^{3 / 2} e^{i \lambda^{1 / 2} Q_{0}+i \pi / 4}\left(3 \lambda^{1 / 2}\right)^{-1 / 3} \sum_{k=0}^{4} \frac{i^{k} A i^{(k)}(0) G_{k}(\tau, t)}{k !\left(3 \lambda^{1 / 2}\right)^{k / 3}}+O\left(\lambda^{-1}\|h\|_{H^{7}}\right)$. 
The coefficients $G_{k}(\tau, t)$ are supported in the interval $\beta<\tau^{-1}+2<\alpha$. For $t_{0} \leq t \leq t_{1}, s>0$, the function $\mathcal{J}_{2}(t, s)$ is $O\left(\lambda^{-1}\|h\|_{H^{2}}\right)$. The remainder constants depend on $A, \beta, t_{0}$ and $t_{1}$.

Proof. First note that the support of $g_{2}(\rho)$ is a compact subinterval of $(0,1)$. It follows from Theorem 2.2 that the remainder term for $\lambda^{1 / 12} \mathcal{W}(\rho)$ is $O\left(\lambda^{-1}\right)$ on $\operatorname{supp}\left(g_{2}\right)$. Now from Theorem 2.2, Lemma 2.3 (8) and Definition 3.1 we have

$$
\begin{aligned}
& \mathcal{J}_{2}(t, s)=(2 \pi)^{1 / 2} \int_{t \text { Imw }=1}\left(\left(\rho^{-2}-1\right)^{-1 / 4} \cos \left(\frac{2}{3} \lambda^{1 / 2}|\zeta|^{3 / 2}-\frac{\pi}{4}\right)\right. \\
& \left.-\frac{\left(3 \rho^{2}+2\right)}{24 \lambda^{1 / 2}\left(\rho^{-2}-1\right)^{1 / 4}\left(1-\rho^{2}\right)^{3 / 2}} \sin \left(\frac{2}{3} \lambda^{1 / 2}|\zeta|^{3 / 2}-\frac{\pi}{4}\right)\right) \\
& \times e^{2 \pi i m \operatorname{Re} A(w)+2 \pi i n \operatorname{Rew}} \tilde{h}(w) g_{2}(\rho) d \operatorname{Rew}+O\left(\|h\|_{H^{0}} \lambda^{-1}\right) .
\end{aligned}
$$

If we express the cosine and sine as a sum of exponentials then following the setup of Section 3 for $\Phi=\epsilon_{*} \frac{2}{3}|\zeta|^{3 / 2}+s R e A(w)+t \operatorname{Rew}, s=2 \pi m \lambda^{-1 / 2}, t=2 \pi n \lambda^{-1 / 2}$, we can write the above integral as

$$
\begin{aligned}
& \sum_{\epsilon_{*}= \pm 1}(\pi / 2)^{1 / 2} e^{-i \epsilon_{*} / 4} \\
& \quad \times \int_{t I m w=1}\left(\rho^{-2}-1\right)^{-1 / 4}\left(1+\frac{i \epsilon_{*}\left(3 \rho^{2}+2\right)}{24 \lambda^{1 / 2}\left(1-\rho^{2}\right)^{3 / 2}}\right) e^{i \lambda^{1 / 2} \Phi} \tilde{h}(w) g_{2}(\rho) d \text { Rew. }
\end{aligned}
$$

We need to understand the support of the integrand. By Proposition 4.1 the product $\rho \tau$ is bounded as $\alpha^{-1}<\rho \tau<\beta^{-1}$ on $\operatorname{supp}(\tilde{h})$, and $\operatorname{supp}\left(g_{2}\right)$ is compact in $(0,1)$. For $\epsilon_{1}=\epsilon_{2}=-1$ the stationary locus $S_{-1}(\tau)$ is separated from $\operatorname{supp}\left(\tilde{h}(w) g_{2}(\rho)\right)$; for $\epsilon_{2}=1, \epsilon_{1}= \pm 1, \Phi$ is non-stationary. For these three cited cases $\frac{d \Phi}{d x}, x=R e w$, is bounded away from zero on $\operatorname{supp}\left(\tilde{h}(w) g_{2}(\rho)\right)$. We can integrate by parts twice with $d u=e^{i \lambda^{1 / 2} \Phi} \frac{d \Phi}{d x} d x$ to show that the integrals are $O\left(\lambda^{-1}\|\tilde{h}\|_{H^{2}}\right)$.

The remaining case is the integral for $\epsilon_{1}=-\epsilon_{2}=1$ (for $t>0, s<0$ and $\left.\epsilon_{*}=-1\right)$. From Proposition 3.3, $\Phi=\Xi^{3}+Q_{0}$ with: $\Xi^{3}=t Q, \Xi$ having a simple zero on $S_{1}(\tau)$ (a segment of $S_{1}(\tau)$ is contained in $\operatorname{supp}\left(\tilde{h}(w) g_{2}(\rho)\right)$ ) and $\Xi$ being an invertible analytic function of $x$. The remaining integral can now be written as

$$
\begin{aligned}
\left(\frac{\pi}{2}\right)^{1 / 2} e^{i \lambda^{1 / 2} Q_{0}+i \pi / 4} \int_{t I m w} & =1 \\
& e^{i \lambda^{1 / 2} \Xi^{3}}\left(\rho^{-2}-1\right)^{-1 / 4} \\
& \times\left(1-\frac{i\left(3 \rho^{2}+2\right)}{24 \lambda^{1 / 2}\left(1-\rho^{2}\right)^{3 / 2}}\right)\left(\frac{d \Xi}{d x}\right)^{-1} \tilde{h}(w) g_{2}(\rho) d \Xi .
\end{aligned}
$$

We will now consider the stationary phase expansion for the integral. As already noted, $\alpha^{-1}<\rho \tau<\beta^{-1}$ on $\operatorname{supp}(\tilde{h})$ and $\operatorname{supp}\left(g_{2}\right)$ is compact in $(0,1)$; and

$$
\left(\rho^{-2}-1\right)^{-1 / 4}\left(1-\frac{i\left(3 \rho^{2}+2\right)}{24 \lambda^{1 / 2}\left(1-\rho^{2}\right)^{3 / 2}}\right)\left(\frac{d \Xi}{d x}\right)^{-1}
$$

is analytic on a neighborhood of $\operatorname{supp}\left(\tilde{h}(w) g_{2}(\rho)\right)$ and thus has bounded derivatives of all orders. We apply Proposition [2.6] to obtain a five-term expansion with a remainder $O\left(\lambda^{-1}\|h\|_{H^{7}}\right)$. 
It remains to calculate the expansion. The stationary phase expansion is given in terms of the $\Xi$-derivatives of

$$
\left(\rho^{-2}-1\right)^{-1 / 4}\left(1-\frac{i\left(3 \rho^{2}+2\right)}{24 \lambda^{1 / 2}\left(1-\rho^{2}\right)^{3 / 2}}\right)\left(\frac{d \Xi}{d x}\right)^{-1} \tilde{h}(w) g_{2}(\rho)
$$

evaluated on the stationary locus $S_{1}(\tau)$. On $\operatorname{supp}(\tilde{h}(w))$ we have $\alpha^{-1}<\rho \tau<\beta^{-1}$; the stationary locus $S_{1}(\tau)=(2 \tau+1)^{-1}$ lies in the region $\alpha^{-1}<\rho \tau<\beta^{-1}$ precisely for $\rho \in\left(1-2 \beta^{-1}, 1-2 \alpha^{-1}\right) ; g_{2}(\rho)$ is identically unity on a neighborhood of $\left[1-2 \beta^{-1}, 1-2 \alpha^{-1}\right]$. The function $g_{2}(\rho)$ can effectively be replaced by unity in calculating the expansion; the expansion terms are given by the $\Xi$-derivatives of

$$
\left(\rho^{-2}-1\right)^{-1 / 4}\left(1-\frac{i\left(3 \rho^{2}+2\right)}{24 \lambda^{1 / 2}\left(1-\rho^{2}\right)^{3 / 2}}\right)\left(\frac{d \Xi}{d x}\right)^{-1} \tilde{h}(w) .
$$

In particular, the support of the expansion terms is contained in $\operatorname{supp}(\tilde{h})$. The parameters $\rho, \tau$ satisfy $\alpha^{-1}<\rho \tau<\beta^{-1}$ and $\rho=(2 \tau+1)^{-1}$; it follows that the expansion terms are supported in the interval $(\alpha-2)^{-1}<\tau<(\beta-2)^{-1}$. Now from (13) and the definition of $\tilde{h}$, we have

$$
\begin{aligned}
& \left(\frac{d \Xi}{d x}\right)^{-1} \tilde{h}(w)=\left(\frac{d \rho}{d x} \frac{d \Xi}{d \rho}\right)^{-1} \tilde{h}(w) \\
& \quad=-\left(2 \rho|t|(\rho \tau(1-\rho \tau))^{1 / 2}\left(\frac{d \Xi}{d \rho}\right)\right)^{-1} h\left(|t|^{-1}\left(\left((\rho \tau)^{-1}-1\right)^{1 / 2}-(\beta-1)^{1 / 2}\right)\right) .
\end{aligned}
$$

The definition and role of $G$ are now established. And finally since $\frac{d}{d \Xi}=\left(\frac{d \Xi}{d \rho}\right)^{-1} \frac{d}{d \rho}$, the proof is complete.

4.6. We are ready to estimate the integral in the region $\rho$ small.

Proposition 4.7. Notation as above. For $t_{0} \leq t \leq t_{1}, s=2 \pi m \lambda^{-1 / 2}$ and $\lambda$ large the function $\mathcal{J}_{3}(t, x)$ is $O\left(\lambda^{-1}|s|^{1 / 2}\|h\|_{H^{2}}\right)$, with the remainder constant depending on $A, \beta, t_{0}$ and $t_{1}$.

Proof. First we look at the remainder terms for the approximation to $\mathcal{K}_{s}$. The support of $g_{3}(\rho)$ is contained in $\left[0,1-2 \beta^{-1}\right)$. From Theorem 2.2 and (8) we have

$$
\begin{gathered}
\lambda^{1 / 12} \mathcal{W}(\rho)=\pi^{-1 / 2}\left(\rho^{-2}-1\right)^{-1 / 4}\left(\cos \left(\frac{2}{3} \lambda^{1 / 2}|\zeta|^{3 / 2}-\frac{\pi}{4}\right)\right. \\
\left.-\frac{\left(3 \rho^{2}+2\right)}{24 \lambda^{1 / 2}\left(1-\rho^{2}\right)^{3 / 2}} \sin \left(\frac{2}{3} \lambda^{1 / 2}|\zeta|^{3 / 2}-\frac{\pi}{4}\right)\right)+O\left(\lambda^{-1} \rho^{1 / 2}\right) .
\end{gathered}
$$

From Proposition 4.1 we have $\rho \leq c_{2}|s|$, and thus the remainder can also be written as $O\left(\lambda^{-1}|s|^{1 / 2}\right)$. Now we have, again in the setup for Section 3, on expressing the cosine as a sum of exponentials,

$$
\begin{gathered}
\mathcal{J}_{3}(t, s)=\sum_{\epsilon_{*}= \pm 1}(\pi / 2)^{1 / 2} e^{-i \epsilon_{*} \pi / 4} \int_{t I m w=1} e^{i \lambda^{1 / 2} \Phi} v(\rho) \tilde{h}(w) g_{3}(\rho) d R e w \\
+O\left(\lambda^{-1}|s|^{1 / 2}\|h\|_{H^{0}}\right)
\end{gathered}
$$

for $v(\rho)=\left(\rho^{-2}-1\right)^{-1 / 4}\left(1+\frac{i \epsilon_{*}\left(3 \rho^{2}+2\right)}{24 \lambda^{1 / 2}\left(1-\rho^{2}\right)^{3 / 2}}\right)$. Since $h$ has compact support, provided $\frac{d \Phi}{d x}$ is bounded away from zero, we can integrate by parts twice with 
$d u=e^{i \lambda^{1 / 2} \Phi} \frac{d \Phi}{d x} d x$ to obtain the new integrand

$$
-\lambda^{-1} e^{i \lambda^{1 / 2} \Phi} \frac{d}{d x}\left(\left(\frac{d \Phi}{d x}\right)^{-1} \frac{d}{d x}\left(\left(\frac{d \Phi}{d x}\right)^{-1} v(\rho) \tilde{h}(w) g_{3}(\rho)\right)\right) d x
$$

for $x=$ Rew. We express the integrand in terms of $\sigma=\rho \tau$ and $\tau$ in order to analyze its magnitude. We have as in Proposition 4.6 that

$$
\tilde{h}(w)=h\left(|t|^{-1}\left(\left(\sigma^{-1}-1\right)^{1 / 2}-(\beta-1)^{1 / 2}\right)\right)
$$

and from Proposition 4.1 that $\alpha^{-1} \leq \sigma \leq \beta^{-1}$ on $\operatorname{supp}(\tilde{h})$. It follows that the product $\tilde{h}(w) g_{3}(\rho)$ is a $C^{k}$-bounded function of $\sigma$ for $\tau$ bounded away from zero. Next observe that

$$
\begin{gathered}
\frac{d}{d x}=\frac{d \sigma}{d x} \frac{d}{d \sigma}=\left(-2|t| \sigma(\sigma(1-\sigma))^{1 / 2}\right) \frac{d}{d \sigma} \\
\frac{d \Phi}{d x}=t q=t\left(1-\epsilon_{1} \sigma \tau^{-1}(1-2 \sigma)+2 \epsilon_{2}\left(\left(1-\sigma^{2} \tau^{-2}\right) \sigma(1-\sigma)\right)^{1 / 2}\right)
\end{gathered}
$$

and

$$
\tau^{1 / 2} v(\rho)=\left(\sigma^{-2}-\tau^{-2}\right)^{-1 / 4}\left(1+\frac{i \epsilon_{*}\left(3 \sigma^{2} \tau^{-2}+2\right)}{24 \lambda^{1 / 2}\left(1-\sigma^{2} \tau^{-2}\right)^{3 / 2}}\right) .
$$

Now from $\operatorname{supp}\left(g_{3}\right) \subset\left[0, \beta(2 \alpha)^{-1}\right)$ and $\alpha^{-1} \leq \sigma \leq \beta^{-1}<1 / 4$ it follows that $2 \beta^{-1} \leq \tau$ and furthermore that $\frac{d}{d x}$, $\frac{d \Phi}{d x}$ and $\tau^{1 / 2} v(\rho)$ are uniformly $C^{k}$-bounded as functions of $\sigma$. For $\tau$ large $q$ is close to $1+2 \epsilon_{2}(\sigma(1-\sigma))^{1 / 2}=\left(\sigma^{1 / 2}+\epsilon_{2}(1-\sigma)^{1 / 2}\right)^{2}$, which is bounded away from zero on $\operatorname{supp}(\tilde{h})$, since $\sigma \leq \beta^{-1}<1 / 4$. For $\tau$ bounded $q$ is bounded away from zero on $\operatorname{supp}(\tilde{h})$, since $\alpha^{-1} \leq \rho \tau \leq \beta^{-1}$ is separated from the stationary locus $S_{\epsilon_{1}}(\tau)$. Now on combining all of the considerations we see that

$$
\frac{d}{d x}\left(\left(\frac{d \Phi}{d x}\right)^{-1} \frac{d}{d x}\left(\left(\frac{d \Phi}{d x}\right)^{-1} v(\rho) \tilde{h}(w) g_{3}(\rho)\right)\right)
$$

is bounded by a multiple of $\tau^{-1 / 2}\|h\|_{C^{2}}$ for $t_{0} \leq|t| \leq t_{1}$, the desired bound, since $\tau^{-1}=|s t| c^{-2}$.

4.7. We are ready to consider the Fourier coefficients of a cuspidal automorphic eigenfunction. Let $\Gamma \subset S L(2 ; \mathbb{R})$ be a cofinite subgroup with a cusp at infinity. We conjugate the group to arrange that the cusp has width one. An eigenform $\psi$ for $\Gamma$ is a $\Gamma$-invariant eigenfunction of the hyperbolic Laplacian with finite $L^{2}(H / \Gamma)$ norm. For $D \psi+\lambda \psi=0, \lambda>1 / 4, \psi$ has a Fourier expansion

$$
\psi(w)=\sum_{m \neq 0} a_{m} y^{1 / 2} K_{i r}(2 \pi|m| y) e^{2 \pi i m x}=\sum_{m \neq 0} a_{m}|2 \pi m|^{-1 / 2} e^{-\pi \lambda^{1 / 2} / 2} \mathcal{K}_{s}(w)
$$

for $w=x+i y, \lambda=\frac{1}{4}+r^{2}, s=2 \pi m \lambda^{-1 / 2}, K_{i r}$ the Macdonald-Bessel function and $\mathcal{K}_{s}$ as in Definition 3.1 (the factor $e^{\pi \lambda^{1 / 2} / 2}$ normalizes the Macdonald-Bessel function).

We review the setup from the previous sections. Let $h(\mu)$ be a smooth function with support in $(0,2)$. Recall the definition of $G_{k}(\tau, t)$ from (19) and of $Q_{0}(t, s)$ from (18). 
Theorem 4.8. Situation as above. Given $0<t_{0}<t_{1}$, choose $\beta>4$ and define $\alpha$ by $(\alpha-1)^{1 / 2}=2 t_{1}+(\beta-1)^{1 / 2}$ Let $s=2 \pi m \lambda^{-1 / 2}, t=2 \pi n \lambda^{-1 / 2}$, and, given $A=\left(\begin{array}{ll}a & b \\ c & d\end{array}\right) \in \Gamma, c>0$, let $\tau=c^{2}|s t|^{-1}$. For $t_{0} \leq t \leq t_{1}$ and $\lambda$ large, we have

$$
\begin{gathered}
\int_{t I m w=1} \psi(w) e^{2 \pi i n R e w} \tilde{h}(w) d R e w \\
=2^{1 / 2} 3^{-1 / 3} \pi^{3 / 2} \lambda^{-5 / 12} \sum_{m<0} a_{m} e^{-\pi \lambda^{1 / 2} / 2}|s|^{-1 / 2} \\
\times e^{i \lambda^{1 / 2} Q_{0}+i \pi / 4} \sum_{k=0}^{4} \frac{i^{k} A i^{(k)}(0) G_{k}(\tau, t)}{\left(3 \lambda^{1 / 2}\right)^{k / 3} k !}+O\left(\lambda^{-3 / 4}\|\psi\|_{2}\|h\|_{H^{7}}\right),
\end{gathered}
$$

where the coefficients $G_{k}(\tau, t)$ are supported in the interval $\beta<\tau^{-1}+2<\alpha$; the remainder constant depends on $A, \beta, t_{0}$ and $t_{1}$.

Proof. We will use the automorphy of $\psi$ to obtain the evaluation for the integral

$$
\mathcal{J}=\int_{t I m w=1} \psi(w) e^{2 \pi i n \operatorname{Rew}} \tilde{h}(w) d \operatorname{Rew} .
$$

In particular, $\psi(w)=\psi(A(w))$, and the integral becomes

$$
\mathcal{J}=\int_{t \operatorname{Im} w=1} \psi(A(w)) e^{2 \pi i n \operatorname{Rew}} \tilde{h}(w) d R e w .
$$

Now from Section 4.2 the functions $\left\{g_{j}\right\}_{j=0}^{3}$ give a partition of unity for $[0, \infty)$, and thus for the integral of an exponential and the Macdonald-Bessel function we have

$$
\int_{\text {tImw }=1} \mathcal{K}_{s}(A(w)) e^{2 \pi i n \operatorname{Rew}} \tilde{h}(w) d \operatorname{Rew}=\sum_{j=0}^{3} \mathcal{J}_{j}(t, s) .
$$

It follows that $\mathcal{J}=\sum_{j=0}^{3} J_{j}$, where $J_{j}=\sum_{m \neq 0} a_{m}|2 \pi m|^{-1 / 2} e^{-\pi \lambda^{1 / 2} / 2} \mathcal{J}_{j}(t, s)$ for $t=$ $2 \pi n \lambda^{-1 / 2}$ and $s=2 \pi m \lambda^{-1 / 2}$.

We proceed and analyze the sums $J_{j}$. Since $|s|$ is comparable to $\rho$ from Proposition 4.1 and $g_{0}$ has support in $(1, \infty]$, we have from Proposition 4.2 the estimate $\left|J_{0}\right| \leq c_{1} \sum_{|m| \geq c_{0} \lambda^{1 / 2}}\left|a_{m}\right||m|^{-3 / 2} e^{-\pi \lambda^{1 / 2} / 2} e^{-c_{2}|m|}$ for appropriate positive constants.

Now from Hecke's trivial estimate $\left|a_{m}\right| \leq \hat{c}|m|^{1 / 2} \lambda^{1 / 4} e^{\pi \lambda^{1 / 2} / 2}\|\psi\|_{2}$ we have that

$$
\left|J_{0}\right| \leq c_{1}^{\prime} \sum_{|m| \geq c_{0} \lambda^{1 / 2}}|m|^{-1} \lambda^{1 / 4} e^{-c_{2}|m|}\|\psi\|_{2}=O\left(e^{-c^{\prime} \lambda^{1 / 2}}\|\psi\|_{2}\right),
$$

a suitable bound for $J_{0}$. Next we consider $J_{1}$ and $J_{3}$. From Propositions 4.5 and 4.7 we have, since $\rho$ is comparable to $|s|$,

$$
\left|J_{1}\right| \leq c_{2} \sum_{c_{0} \lambda^{1 / 2} \leq|m| \leq c_{1} \lambda^{1 / 2}}\left|a_{m}\right||m|^{-1 / 2} \lambda^{-1} e^{-\pi \lambda^{1 / 2} / 2}
$$

and

$$
\left|J_{3}\right| \leq c_{4} \sum_{|m| \leq c_{3} \lambda^{1 / 2}}\left|a_{m}\right| \lambda^{-5 / 4} e^{-\pi \lambda^{1 / 2} / 2}
$$


for appropriate positive constants. The next step is to apply Hölder's inequality. We find that $\left|J_{1}\right|$ is bounded in terms of

$$
\lambda^{-1}\left(\sum_{c_{0} \lambda^{1 / 2} \leq|m| \leq c_{1} \lambda^{1 / 2}}\left|a_{m}\right|^{2} e^{-\pi \lambda^{1 / 2}}\right)^{1 / 2}\left(\sum_{c_{0} \lambda^{1 / 2} \leq|m| \leq c_{1} \lambda^{1 / 2}}|m|^{-1}\right)^{1 / 2},
$$

which from Lemma 5.1 is $O\left(\lambda^{-3 / 4}\|\psi\|_{2}\right)$, the desired bound for $J_{1}$. In the same fashion $\left|J_{3}\right|$ is bounded in terms of

$$
\lambda^{-1}\left(\sum_{|m| \leq c_{3} \lambda^{1 / 2}}\left|a_{m}\right|^{2} e^{-\pi \lambda^{1 / 2}}\right)^{1 / 2} .
$$

From Lemma 5.1 it follows that $\left|J_{3}\right|$ is also $O\left(\lambda^{-3 / 4}\|\psi\|_{2}\right)$, the desired bound for $J_{3}$.

We proceed and consider $J_{2}$. First we express the result of Proposition 4.6 in the form $\mathcal{J}_{2}(t, s)=\mathcal{G}(t, s)+O\left(\lambda^{-1}\right)$, where $\mathcal{G}$ is the explicit sum. The contribution to $J_{2}$ from the remainder term is $O\left(\lambda^{-1} \sum_{c_{0} \lambda^{1 / 2} \leq|m| \leq c_{1} \lambda^{1 / 2}}\left|a_{m}\right||m|^{-1 / 2} e^{-\pi \lambda^{1 / 2} / 2}\right)$, where the summation range is again determined from Proposition 4.6. The sum has the same form as the estimating sum for $J_{1}$. The contribution from the remainder term to $J_{2}$ is $O\left(\lambda^{-3 / 4}\|\psi\|_{2}\right)$. The expansion for the integral $\mathcal{J}$ is established with the summation range given in Proposition 4.6.

Corollary 4.9. Situation as above. For $s=2 \pi m \lambda^{-1 / 2}, t=2 \pi n \lambda^{-1 / 2}, t_{0} \leq t \leq t_{1}$ and $\lambda$ large, the Fourier coefficients of the automorphic eigenfunction $\psi$ satisfy the following:

$$
\begin{gathered}
a_{n} e^{-\pi \lambda^{1 / 2} / 2}=2^{1 / 6} 3^{-1 / 3} \pi^{1 / 2} \lambda^{-1 / 4}\left(A i(0)+\frac{2^{1 / 3}}{70 \lambda^{2 / 3}} A i^{(1)}(0)\right)^{-1} \\
\times \sum_{m>0} a_{m} e^{-\pi \lambda^{1 / 2} / 2} s^{-1 / 2} t^{1 / 2} e^{-i \lambda^{1 / 2} Q_{0}-i \pi / 4} \sum_{k=0}^{4} \frac{A i^{(k)}(0) \overline{G_{k}(\tau, t)}}{i^{k}\left(3 \lambda^{1 / 2}\right)^{k / 3} k !} \\
+O\left(\lambda^{-7 / 12}\|\psi\|_{2}\|h\|_{H^{7}}\right),
\end{gathered}
$$

where the coefficients $G_{k}(\tau, t)$ are supported in the interval $\beta<\tau^{-1}+2<\alpha$. The Fourier coefficients $a_{n}, t_{0} \leq t \leq t_{1}$, are bounded as $O\left(\lambda^{1 / 4}\|\psi\|_{2}\|h\|_{H^{7}}\right)$. The remainder constants depend on $A, \beta, t_{0}$ and $t_{1}$.

Proof. The coefficient relation will result from equating two evaluations of the integral

$$
\mathcal{J}=\int_{t \operatorname{Im} w=1} \psi(w) e^{2 \pi i n \operatorname{Rew}} \tilde{h}(w) d \operatorname{Rew}
$$

for $t=2 \pi n \lambda^{-1 / 2}>0$. First note that since $\psi(w) e^{2 \pi i n R e w}$ is unit-translation invariant, the integral can be replaced by an integral over $0 \leq$ Rew $\leq 1$, provided $\tilde{h}(w)$ is replaced by the sum $\sum_{k \in \mathbf{Z}} \tilde{h}(w+k)$. Provided $h$ satisfies the summation condition $\sum_{k \in \mathbb{Z}} h(\mu+k) \equiv 1$, then by the orthogonality of exponentials, Definition 3.1, and Lemma 2.3 the integral is simply $\bar{a}_{n} 2^{1 / 2} \pi t^{-1 / 2} \lambda^{-1 / 6} e^{-\pi \lambda^{1 / 2} / 2} \mathcal{W}(1)\left(1+O\left(\lambda^{-1}\right)\right)$. The expansions of Chapter 2 can be combined to show that

$$
\mathcal{W}(1)=2^{-1 / 6}\left(A i(0)+\lambda^{-2 / 3} B_{0}(0) A i^{\prime}(0)\right)+O\left(\lambda^{-1}\right)
$$


and so for $n>0$, after noting that $B_{0}(0)=2^{1 / 3} / 70$, we find that

$$
\mathcal{J}=\bar{a}_{n} 2^{1 / 3} \pi t^{-1 / 2} \lambda^{-1 / 6} e^{-\pi \lambda^{1 / 2} / 2}\left(A i(0)+\lambda^{-2 / 3} 2^{1 / 3} A i^{\prime}(0) / 70+O\left(\lambda^{-1}\right)\right),
$$

the desired first evaluation of the integral.

The second evaluation of the integral is given in Theorem 4.8. The coefficient relation is the consequence of equating the two evaluations. The coefficient bound is a further consequence of Lemma 5.1 .

We consider the evaluation of $\frac{d \Xi}{d \rho}$ and the first term of $G_{0}$. We start by evaluating $\frac{d^{3} Q}{d \kappa^{3}}$ on $S_{1}(\tau)$. From (17) observe that for $t>0$ and $\epsilon_{1}=-\epsilon_{2}=1$, we have

$$
\left.\frac{d^{3} Q}{d \kappa^{3}}\right|_{S_{1}}=-\left.\left(\rho t(\rho \tau(1-\rho \tau))^{1 / 2}\right)^{-1} \frac{d}{d \rho}(\mathcal{A}-\mathcal{B})\right|_{S_{1}} .
$$

We further find that

$$
\frac{d \mathcal{A}}{d \rho}=((1+\rho) \rho \tau)^{-1 / 2}(2 \rho \tau+\tau) / 2
$$

and

$$
\frac{d \mathcal{B}}{d \rho}=((1-\rho \tau)(1-\rho))^{-1 / 2}(2 \rho \tau-\tau-1) / 2 .
$$

For $\rho=S_{1}(\tau)$ we combine the formulas and find that

$$
\left.\frac{d^{3} t Q}{d \kappa^{3}}\right|_{S_{1}}=-2^{-3 / 2}(2 \tau+1)^{4}\left(\tau^{2}+\tau\right)^{-1}
$$

Furthermore, for $\rho=S_{1}(\tau)$ we have

$$
\frac{d \Xi}{d \rho}=\left(\frac{1}{6} \frac{d^{3} t Q}{d \kappa^{3}}\right)^{1 / 3}=-6^{-1 / 3} 2^{-1 / 2}(2 \tau+1)^{4 / 3}\left(\tau^{2}+\tau\right)^{-1 / 3} .
$$

Now from (19) we have for the cutoff function $h$ that the first term of $G_{0}$ is

$$
\begin{aligned}
& -\left.\left(2 \rho t(\rho \tau(1-\rho \tau))^{1 / 2}\left(\rho^{-2}-1\right)^{1 / 4} \frac{d \Xi}{d \rho}\right)^{-1} h\right|_{S_{1}(\tau)} \\
& =-\left.\left(2^{3 / 2} t(2 \tau+1)^{-2}\left(\tau^{2}+\tau\right)^{3 / 4} \frac{d \Xi}{d \rho}\right)^{-1} h\right|_{S_{1}(\tau)} \\
& =\left.2^{-1} 6^{1 / 3} t^{-1}(2 \tau+1)^{2 / 3}\left(\tau^{2}+\tau\right)^{-5 / 12} h\right|_{S_{1}(\tau)} .
\end{aligned}
$$

We consider the nature of the coefficient relation. The relation has the general form of a matrix equation $\mathcal{T}=\mathcal{R S}$ for $\mathcal{T}$, respectively $\mathcal{S}$, the transpose vector of the coefficient sequence $\left(a_{n}\right)$ for $t_{0} \leq 2 \pi n \lambda^{-1 / 2} \leq t_{1}$, respectively for $s_{0} \leq 2 \pi m \lambda^{-1 / 2} \leq$ $s_{1}$, and $\mathcal{R}$ the matrix $\left(g(t, s) \lambda^{-1 / 4} e^{-i \lambda^{1 / 2} Q_{0}(t, s)}\right)$. The nature of $\mathcal{R}$ is suggested by considering the matrix $\left(\lambda^{-1 / 4} e^{-i \lambda^{1 / 2} Q_{0}(t, s)}\right)$ with the parameter $t$ indexing the row vector $\left(\lambda^{-1 / 4} e^{-i \lambda^{1 / 2} Q_{0}(t, s)}\right), s_{0} \leq s \leq s_{1}$. The row vectors have Euclidean norm $\sim\left((2 \pi)^{-1}\left(s_{1}-s_{0}\right)\right)^{1 / 2}$. The Hermitian pairing of the index $t^{\prime}$ and index $t$ rows is given by the exponential sum $\lambda^{-1 / 2} \sum_{s_{0} \leq s \leq s_{1}} e^{-i \lambda^{1 / 2}\left(Q_{0}\left(t^{\prime}, s\right)-Q_{0}(t, s)\right)}$. The behavior of the sum is foreshadowed by the derivative of the phase with respect to the summation parameter $m$. In particular for $s=2 \pi m \lambda^{-1 / 2}$ we have that

$$
(2 \pi)^{-1} \lambda^{1 / 2} \frac{d Q_{0}}{d m}=s^{-1}\left(1+\tau^{-1}\right)^{1 / 2}-a c^{-1}
$$


and

$$
(2 \pi)^{-1} \lambda^{1 / 2} \frac{d}{d m}\left(Q_{0}\left(t^{\prime}, s\right)-Q_{0}(t, s)\right)=s^{-1}\left(\left(1+\sigma^{-1}\right)^{1 / 2}-\left(1+\tau^{-1}\right)^{1 / 2}\right)
$$

for $\sigma^{-1}=t^{\prime} s c^{-2}$ and $\tau^{-1}=t s c^{-2}$. Now in Corollary 4.9 for a given $t$-range and parameter $\beta$ the parameter $\alpha$ is determined and the $s$-range is determined by $\beta<\tau^{-1}+2<\alpha+2$ or equivalently by $c^{2} t^{-1}(\beta-2)<s<c^{2} t^{-1}(\alpha-2)$. It follows that $\left(1+\sigma^{-1}\right)^{1 / 2}$ and $\left(1+\tau^{-1}\right)^{1 / 2}$ are bounded, and furthermore for $c$ large that $s_{1}-s_{0}$ is large while the indicated derivative is small. It is also necessary to consider the convexity properties of $Q_{0}$. The first derivative

$$
(2 \pi)^{-1} \lambda^{1 / 2} \frac{d Q_{0}}{d m}=\left(s^{-2}+s^{-1} t c^{-2}\right)^{1 / 2}-a c^{-1}
$$

is an increasing function of $s^{-1}$ with the rate of increase increasing with $t$. The phase $Q_{0}\left(t^{\prime}, s\right)-Q_{0}(t, s)$ is convex in $s$ for $t^{\prime}>t$. We recognize that there are two situations to consider for $\lambda$ tending to infinity. First, for the difference $t^{\prime}-t$ tending to zero the exponential sum will exhibit the behavior of a stationary-phase sum [Tm, Chap. 4] and is expected to have magnitude $O\left(\lambda^{-1 / 4}\right)$. Second, for the difference $t^{\prime}-t$ bounded away from zero and for an appropriate choice of the parameter $c$ the sum is found to be bounded by $O\left(\lambda^{-1 / 2}\right)$ on applying the van der Corput lemma Tm, Chap. 4]. The rows of $\mathcal{R}$ become pairwise orthogonal, and it appears that a consecutive sequence of rows spans a parallelepiped with volume tending to zero.

4.8. We are ready to consider an application of our results to integrals of eigenfunctions along vertical lines in the upper half plane. Let $\Gamma \subset S L(2 ; \mathbb{R})$ be a cofinite subgroup with a width one cusp at infinity, and $\psi$ a $\Gamma$-invariant eigenfunction with finite $L^{2}(H / \Gamma)$ norm. For $\chi \in C_{0}^{\infty}(H), \gamma$ a complete geodesic on $H$, and $d s$ the hyperbolic arc length element, consider the Radon integral

$$
\mathcal{R}(\psi \chi ; \gamma)=\int_{\gamma} \psi \chi d s,[\mathrm{Hl}] .
$$

For the vertical geodesics Proposition 2.7 provides an expansion for $\mathcal{R}$.

Theorem 4.10. Situation as above. For an eigenform $\psi$ with eigenvalue $\lambda$ and Fourier expansion (4.2), and for $g(y)$ a smooth function with support a compact subinterval of $(0, \infty)$, we have

$$
\begin{aligned}
& \int_{0}^{\infty} \psi(x+i y) g(y) y^{-1} d y \\
& =\pi \lambda^{-1 / 4} e^{-\pi \lambda^{1 / 2} / 2} \sum_{m} a_{m}|2 \pi m|^{-1 / 2} e^{2 \pi i m x} g\left(|2 \pi m|^{-1} \lambda^{1 / 2}\right) \\
& \quad+O_{g}\left(\lambda^{-3 / 4} \log \lambda\|\psi\|_{2}\right) .
\end{aligned}
$$

Proof. The considerations are based on substituting the Fourier expansion (20) for $\psi$ and analyzing the resulting integrals. In the case $|2 \pi m| \geq c_{0} \lambda^{1 / 2}$ by Proposition 2.7 the Macdonald-Bessel integrals are $O\left(e^{-c_{1}|m|}\right)$ for a positive constant. Then by Hecke's trivial coefficient estimate the sum of such terms is bounded by a multiple of $\sum_{|m| \geq c_{0} \lambda^{1 / 2}}|m|^{1 / 2} \lambda^{1 / 4} e^{-c_{1}|m|}\|\psi\|_{2}=O\left(e^{-c^{\prime} \lambda^{1 / 2}}\|\psi\|_{2}\right)$, a suitable bound for 
the sum. The next consideration is the contribution to the remaining sum of the remainder term from Proposition 2.7] The contribution is bounded in terms of

$$
\lambda^{-1} \sum_{|m| \leq c_{0} \lambda^{1 / 2}}\left|a_{m}\right| e^{-\lambda^{1 / 2} / 2}|2 \pi m|^{-1 / 2}\|\psi\|_{2} .
$$

We apply Hölder's inequality to find the bound $O\left(\lambda^{-3 / 4} \log \lambda\|\psi\|_{2}\right)$. The desired expansion now follows from Proposition 2.7.

\section{APpendix}

Let $\Gamma$ be a cofinite group with a width one cusp at infinity. A fundamental domain $\mathcal{F}$ is standard for a cusp $\hat{c}$ for $\Gamma$ provided that for $C \in \Gamma$ generating the

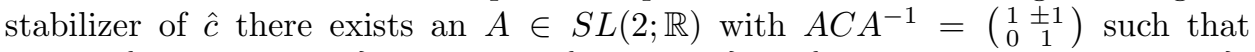
$A \mathcal{F} \subset\{0 \leq \operatorname{Rew} \leq 1\}$ and $A \mathcal{F} \cap\{\operatorname{Imw} \geq 1\}=\{0 \leq \operatorname{Rew}<1$, Imw $\geq 1\}$. Introduce the rectangle $\operatorname{Rect}\left(y_{0}\right)=\left\{0<\operatorname{Rew}<1\right.$, Imw $\left.>y_{0}\right\}$ and the counting function $\mathcal{N}_{\Gamma}\left(y_{0}\right)=\#\left\{A \in \Gamma \mid A \mathcal{F} \cap \operatorname{Rect}\left(y_{0}\right) \neq \emptyset\right\}$. We present a refinement of the estimate of Deshouillers and Iwaniec [DI, Corollary 1, pg. 62]. Let $\psi$ be a cuspidal automorphic eigenfunction with Fourier expansion (20).

Lemma 5.1. Notation as above. There exists a positive constant $C_{\Gamma}$ such that, for $\lambda$ sufficiently large and all positive integers $M$,

$$
\sum_{|m| \leq M}\left|a_{m}\right|^{2} e^{-\pi \lambda^{1 / 2}} \leq C_{\Gamma}\left(M+\lambda^{1 / 2}\right)\|\psi\|_{2}^{2}
$$

Proof. The approach is to estimate the integral of $\psi^{2}$ over $\mathcal{R}=\operatorname{Rect}\left(\lambda^{1 / 2}(4 \pi M)^{-1}\right)$. First we integrate in $x$, use orthogonality of exponentials, and take a partial sum as a lower bound, to find that

$$
\int_{\mathcal{R}} \psi^{2} d \mathcal{A} \geq \sum_{|m| \leq M}\left|a_{m}\right|^{2} \int_{\lambda^{1 / 2}(8 \pi M)^{-1}}^{\infty}\left(K_{i r}(2 \pi|m| y)\right)^{2} y^{-1} d y .
$$

The $y$-integrals are bounded below by $C \lambda^{-1 / 2} e^{-\pi \lambda^{1 / 2}}$ from Corollary [2.5] Now, since $\psi$ is $\Gamma$-invariant, $\int_{\mathcal{R}} \psi^{2} \mathrm{~d} \mathcal{A}$ is bounded by the product $\|\psi\|_{2}^{2} \mathcal{N}_{\Gamma}\left(\lambda^{1 / 2}(4 \pi M)^{-1}\right)$. By Lemma 5.2 (proved below), $\mathcal{N}_{\Gamma}\left(\lambda^{1 / 2}(4 \pi M)^{-1}\right)$ is bounded by $C_{\Gamma}\left(\lambda^{-1 / 2} M+1\right)$. The desired estimate follows on combining inequalities.

We wish to introduce a decomposition of a fundamental domain $\mathcal{F}$ that is standard for all of its cusps. Suppose as above that $\mathcal{F}$ is standard for the cusp $\hat{c}$ with stabilizer generated by $C$ and $A C A^{-1}=\left(\begin{array}{cc}1 & \pm 1 \\ 0 & 1\end{array}\right)$. Define the cuspidal neighborhood $\mathcal{F}_{\epsilon}=A^{-1}\{0 \leq \operatorname{Re} z<1$, Imz $\geq 2\}$ and the cuspidal region $\mathcal{F}_{\mathrm{CUSP}}=\bigcup_{\hat{c}_{j}} \mathcal{F}_{\hat{c}_{j}}$. Further define $\mathcal{F}_{\text {THICK }}=\mathcal{F}-\mathcal{F}_{\text {CUSP }} . \mathcal{F}_{\text {THICK }}$ is relatively compact, thus has finite diameter $\delta$. We are ready to prove the following.

Lemma 5.2. Notation as above. There exists a positive constant $C_{\Gamma}$ such that

$$
\mathcal{N}_{\Gamma}\left(y_{0}\right) \leq C_{\Gamma}\left(y_{0}^{-1}+1\right) .
$$

Proof. The issue is to count the number of translates of $\mathcal{F}$ intersecting $\operatorname{Rect}\left(y_{0}\right)$. First count the number of translates of $\mathcal{F}_{\text {THICK }}$ intersecting $\operatorname{Rect}\left(y_{0}\right)$. Observe that if a translate intersects $\operatorname{Rect}\left(y_{0}\right)$, then it is contained in $\operatorname{Rect}\left(y_{0} e^{-2 \delta}\right)$, since points of $\operatorname{Rect}\left(y_{0} e^{-2 \delta}\right)^{c}$ are at least $2 \delta$ away from $\operatorname{Rect}\left(y_{0}\right)$. Thus, by considering 
areas, $\operatorname{Rect}\left(y_{0} e^{-2 \delta}\right)$ contains at most $C_{\mathcal{F}} y_{0}^{-1}$ complete translates of $\mathcal{F}_{\text {THICK}}$; at most $C_{\mathcal{F}} y_{0}^{-1}$ translates of $\mathcal{F}_{\text {THICK }}$ intersect $\operatorname{Rect}\left(y_{0}\right)$.

It remains to count the number of translates of $\mathcal{F}_{\text {CUSP }}$ intersecting $\operatorname{Rect}\left(y_{0}\right)$. The first step will be to count horocycles. Every horocycle bounds a horodisc HD. We will refer to horocycles for cusps of $\Gamma$ by the area of the quotient $H D / \Gamma$. It is standard that the area one horocycles for distinct cusps on $H$ of $\Gamma$ are disjoint. In fact the area $\frac{1}{2}$ horocycles for distinct cusps on $H$ of $\Gamma$ are uniformly separated. A curve connecting two area $\frac{1}{2}$ horocycles has length at least twice the distance from an area $\frac{1}{2}$ to an area 1 horocycle, at least $2 \log 2$. Fix $\rho$ positive, $\rho<\log 2$.

For each area $\frac{1}{2}$ horocycle $h$ on $H$ of a cusp of $\Gamma$ (other than infinity), consider the metric disc $D_{h}$ with radius $\rho$ and center the highest point of $h$ (with maximal imaginary part). We are ready to use the discs to count the number of area $\frac{1}{2}$ horocycles intersecting a given $\operatorname{Rect}\left(y_{0}\right)$. Each area $\frac{1}{2}$ horocycle (other than infinity) is disjoint from the area 1 horodisc at infinity $\{I m w \geq 1\}$; the center of each $D_{h}$ lies below $\{$ Imw $=1\}$. Decreasing $\rho$ if necessary, we can arrange that if $D_{h} \cap$ $\{0<$ Rew $<1\} \neq \emptyset$, then $D_{h} \subset\{-1<$ Rew $<2\}$. Thus, for an area $\frac{1}{2}$ horocycle, if $D_{h} \cap \operatorname{Rect}\left(y_{0}\right) \neq \emptyset$, then $D_{h} \subset\left\{-1<\operatorname{Rew}<2\right.$, Imw $\left.>y_{0} e^{-\rho}\right\}$. By considering areas we have that the number of such horocycles is bounded by

$$
\operatorname{Area}\left(\left\{-1<\operatorname{Rew}<2, \operatorname{Imw}>y_{0} e^{-\rho}\right\}\right)\left(\operatorname{Area}\left(D_{h}\right)\right)^{-1}=C_{\mathcal{F}} y_{0}^{-1},
$$

the desired bound for area $\frac{1}{2}$ horocycles intersecting $\operatorname{Rect}\left(y_{0}\right)$.

Finally we consider the count for $\operatorname{Rect}\left(y_{0}\right)$ intersecting a given horocycle. Consider $\operatorname{Rect}\left(y_{0}\right)$ and an intersected horocycle $h$ with highest point $h_{*}$, and let $d=$ $\operatorname{dist}\left(\partial \operatorname{Rect}\left(y_{0}\right), h_{*}\right)$. By an auxiliary transformation $B$, conjugate $\Gamma$ and map $h$ to the height two horocycle (area $\frac{1}{2}$ ) at infinity and $\partial \operatorname{Rect}\left(y_{0}\right)$ to a horocycle at the origin. The horocycle at the origin has highest point $\left(0,2 e^{d}\right)$ (at distance $d$ from $\{I m w=2\})$. The horocycle at the origin has Cartesian equation $x^{2}+y^{2}-2 e^{d} y=0$ and intersects the height two line at $\left( \pm 2\left(e^{d}-1\right)^{1 / 2}, 2\right)$ Now the stabilizer in $B \Gamma B^{-1}$ of the cusp at infinity is generated by a unit translation. Thus the horocycle at the origin intersects at most $4\left(e^{d}-1\right)^{1 / 2}+2$ translates of $B \mathcal{F}$ above height two (i.e. $\operatorname{Rect}\left(y_{0}\right)$ intersects at most $4\left(e^{d}-1\right)^{1 / 2}+2$ translates of $\mathcal{F}$ inside $h$ ), the desired estimate.

We are ready to combine our estimates and obtain the overall count. Let $\mathbf{N}\left(y_{0}\right)$ be the counting function for the number of area $\frac{1}{2}$ horocycles of $\Gamma$ (other than infinity) intersecting $\operatorname{Rect}\left(y_{0}\right)$; we found that $\int_{y_{0}}^{1}-d \mathbf{N}(y) \leq C_{\mathcal{F}} y_{0}^{-1}(d \mathbf{N}$ is negative). The total number of translates of $\mathcal{F}_{\mathrm{CUSP}}$ intersecting $\operatorname{Rect}\left(y_{0}\right)$ is bounded by counting translates in a given horodisc and then counting distinct horodiscs. Thus a bound is $1-\int_{y_{0}}^{1}\left(4\left(e^{d}-1\right)^{1 / 2}+2\right) d \mathbf{N}(y)$, for $e^{d}=y y_{0}^{-1}$. Integration by parts gives

$$
1+2 \mathbf{N}\left(y_{0}\right)+y_{0}^{-1} \int_{y_{0}}^{1} 2\left(y y_{0}^{-1}-1\right)^{-1 / 2} \mathbf{N}(y) d y,
$$

and using the inequality $\mathbf{N}(y) \leq C_{\mathcal{F}} y^{-1}$ and the substitution $\tau=y y_{0}^{-1}$ for the integral gives

$$
1+2 C_{\mathcal{F}} y_{0}^{-1}\left(1+\int_{1}^{y_{0}^{-1}}(\tau-1)^{-1 / 2} \tau^{-1} d \tau\right) \leq 1+C_{\mathcal{F}}^{\prime} y_{0}^{-1}
$$


In summary, the counts for $\mathcal{F}_{\text {THICK }}$ and $\mathcal{F}_{\text {CUSP }}$ are each bounded by multiples of $\left(y_{0}^{-1}+1\right)$, as desired.

\section{REFERENCES}

[Bl] C.B. Balogh, Asymptotic expansions of the modified Bessel function of the third kind of imaginary order, SIAM J. Appl. Math. 15(1967), 1315-1323. MR 36:5406

[Bm] D. Bump, The Rankin-Selberg method: A survey, in Number Theory, Trace Formulas and Discrete Groups, K.E. Aubert et al., eds., Academic Press, San Diego, 1989, 49-109. MR 90m:11079]

[BDHI] D. Bump, W. Duke, J. Hoffstein and H. Iwaniec, An estimate for the Hecke eigenvalues of Maass forms, Duke Math. J. Research Notices 4 (1992), 75-81. MR 93d:11047

[DI] J.M. Deshouillers and H. Iwaniec, The non-vanishing of Rankin-Selberg zeta functions at special points, Selberg Trace Formula and Related Topics, Contemp. Math. 53(1986), 59-95. MR 88d:11047

[GSh] S. Gelbart and F. Shahidi, Analytic properties of automorphic L-functions, Academic Press, Boston, 1988. MR 89f:11077

[GS] A. Grigis and J. Sjöstrand, Microlocal Analysis for Differential Operators, L.M.S. Lecture Note Series 196, Cambridge University Press, 1994. MR 95d:35009

[HA] D.A. Hejhal and S. Arno, On Fourier coefficients of Maass waveforms for PSL $(2, \mathbb{Z})$, Univ. of Minnesota Supercomputer Institute Research Report 1992/20, 72pp; Math. Comp. 61(1993), 245-267 and S11-S16. MR 94a:11062

[Hj1] D.A. Hejhal, The Selberg Trace Formula for PSL $(2 ; \mathbb{R})$, Vol. 2, Lecture Notes in Math. 1001, Springer-Verlag, 1983. MR 86e:11040

[Hj2] D.A. Hejhal, Eigenvalues of the Laplacian for Hecke Triangle Groups, Memoirs 469 AMS, Providence, 1992. MR 93f:11043

[Hl] S. Helgason, Geometric Analsysis on Symmetric Spaces, Surveys and Monographs 39, AMS, Providence, 1994. MR 96h:43009

[Iw1] H. Iwaniec, Spectral theory of automorphic functions and recent developments in analytic number theory, Proc. I. C. M., Berkeley, 1986, pp. 444-456. MR 89k:11040

[Iw2] H. Iwaniec, Introduction to the Spectral Theory of Automorphic Forms, Revista Matemática Iberoamericana, Madrid, 1995. MR 96f:11078

[Lb] N.N. Lebedev, Special Functions and Their Applications, Dover, New York, 1972. MR 50:2568

[Ms] H. Maass, Über eine neue Art von nichtanalytischen automorphen Funktionen und die Bestimmung Dirichletscher Reihen durch Funktionalgleichungen, Math. Ann. 121(1949), 141-183. MR 11:163c

[Ov] F.W.J. Olver, Asymptotics and Special Functions, Academic Press, San Diego, 1974. MR 55:8655

[Pt1] Y.N. Petridis, On squares of eigenfunctions for the hyperbolic plane and a new bound on certain $L$-series, Internat. Math. Res. Notices (1995), 111-127. MR 96d:11058

[Pt2] Y.N. Petridis, Fourier coefficients of cusp forms, in Number theory (Ottawa, ON, 1996), CRM Proc. Lecture Notes, vol. 19, Amer. Math. Soc., Providence, RI, 1999, 297-307. MR 2000c: 11070

[Sr1] P. Sarnak, Arithmetic Quantum Chaos, in The Schur Lectures 1992, I. Piatetsky-Shapiro and S. Gelbart, eds., IMCP 8 (1995), 183-236. MR 96d:11059

[Sr2] P. Sarnak, Integrals of products of eigenfunctions, Internat. Math. Res. Notices (1994), 251-260. MR 95i:11039

[Sb1] A. Selberg, Harmonic analysis and discontinuous groups in weakly symmetric riemannian spaces with applications to Dirichlet series, J. Indian Math. Soc. 20(1956), 47-87. MR 19:531g

[Sb2] A. Selberg, On the estimation of Fourier coefficients of modular forms, Proc. Symp. Pure Math., vol. 8, Amer. Math. Soc., (1965), 1-15. MR 32:93

[Tr] A. Terras, Harmonic Analysis on Symmetric Spaces and Applications, I, Springer Verlag, New York, 1985. MR 87f:22010

[Tm] E.C. Titchmarsh, The Theory of the Riemann Zeta-function, Oxford University Press, Oxford, 1986. MR 88c:11049 
[Vk] A.B. Venkov, Spectral theory of automorphic functions and its applications, Kluwer Academic, Dordrecht, 1990. MR 93a:11046

[Wp] S.A. Wolpert, Semiclassical limits for the hyperbolic plane, Duke Math. J. 108(2001), 449-509. MR 2003b:11051

3400 AV Williams Building, University of Maryland, College Park, Maryland 20742

E-mail address: saw@math.umd.edu 